\title{
Quantum Tic-Tac-Toe, Spooky-Coins \& Magic-Envelopes, as Metaphors for Relativistic Quantum Physics
}

\author{
Allan Goff, AIAA Member, President, Novatia, Folsom, California* \\ Dale Lehmann, AIAA Member, Software Engineer, DST Output, El Dorado Hills, California \\ Dr. Joel Siegel, AIAA Member, Professor, Sierra Community College, Rocklin, California
}

\begin{abstract}
Two metaphors for quantum physics and nonlocality are presented, one in the form of a game, Quantum TicTac-Toe, and the other as an extended version of Spooky-Coins \& Magic-Envelopes. Quantum Tic-TacToe introduces the concept of quantum games as metarules on classical games, such that states in the quantum game imply the existence of multiple instances of classical games in simultaneous play. Further quantum mechanical implications of these rules will be discussed including a measurement mechanism, the correspondence principle, Everett's Many Worlds Hypothesis, and spooky action at a distance. It also shows the consistency of backwards-in-time causality, an ascertainity principle, and the influence of both futures and pasts that never happened on the present. The Spooky-Coins $\&$ Magic-Envelopes metaphor shows how the two basic rules of quantum games (mixed states and quantum entanglement) can utilize spooky action at a distance to send information faster than light. Relativistic effects are introduced into the metaphor, which permit backwards in time signaling and temporal paradox. Although temporal paradox is not resolvable within the metaphor it appears to be so in reality. Possible implications of a paradigm shift from the current machine metaphor of reality to a game metaphor are briefly considered.
\end{abstract}

\section{INTRODUCTION}

This paper presents two metaphors for quantum physics and nonlocality. They are Quantum Tic-Tac-Toe, and an extended version of Spooky-Coins \& MagicEnvelopes. A brief background of the basic issues is first presented.

The next section presents the first metaphor, Quantum Tic-Tac-Toe. This game is a variation of Classical TicTac-Toe where every quantum move consists of a superposition of two moves in the classical game and self-

'*Copyright $₫ 2002$ by Novatia, Inc. Published by the American Institute of Aeronautics and Astronautics, Inc. with permission. reference is introduced as a measurement mechanism. The intent behind creating the game, the rules, and the mapping of quantum physics are all covered.

In the next section a playful metaphor for quantum nonlocality is introduced, Spooky-Coins and MagicEnvelopes, which is extended to cover the EPR experiment, Bell's theorem, and our FTL protocol. Another look is directed at self-reference as a measurement mechanism, as is the ability of the inclusion of relativistic effects to create temporal paradox.

The final section takes a look at some of the implications of the metaphors, for both science and philosophy, and lays out some of the areas for future research.

\section{BACKGROUND}

One of the great challenges for physicists over the last 100 years has been to accept the changing world view required by relativity and quantum mechanics. Despite the spectacular success of both theories individually there are fundamental inconsistencies between them. Furthermore, the philosophical and physical implications of quantum mechanics have yet to be determined. Physicists still struggle with the interpretation and implications of quantum mechanics. It is to the above issues that this talk will be addressed.

There remain two fundamental unsolved problems in quantum mechanics: relativistic consistency and the measurement process.

In the current formalism, once a measurement occurs, the wave function instantly collapses, going to unity for the measured value, and to zero everywhere else. The problem with this is that "instantaneous" is not a relativistic concept. Simultaneity is not a relativistic invariant. Different relativistic observers cannot agree on when the collapse occurs, or even which measurement caused it. This has been a tolerable problem only because the randomness of quantum phenomena prevents any measurable differences from existing between the different frames-of-reference. 
While the rules of thumb about what constitutes a measurement work as a practical matter, formal definitions have been hard to come by. Unfortunately, we operate at the level of, "I know a measurement when I see one."

John Bell is adamant about the ultimate significance of this shortcoming ${ }^{1}$,

"These considerations, in our opinion, lead inescapably to the conclusion that quantum mechanics is, at the best, incomplete. We look forward to a new theory which can refer meaningfully to events in a given system without requiring 'observation' by another system."

\section{QUantum TIC-TaC-ToE}

Tic-Tac-Toe is a classic children's game that reinforces our prejudices toward a classical reality. It is played on a board with fixed space and time elements, with pieces that are distinct, isolated, and clearly located. There are few games either simpler or more intuitive.

\section{The Intent}

Quantum Tic-Tac-Toe was invented for two purposes; to communicate elements of the quantum character of nature, and to make clear some key concepts in a protocol that uses quantum nonlocality to break the speed of light. The latter is discussed in a companion paper ${ }^{2}$, while the former will be discussed presently.

It is no secret that the transition from classical to quantum mechanical thought is a challenge - one that some students regrettably fail to make. Games, however, are very accessible and come with few preconceptions. All the rules are written down and made available before the start of play. In nature we can only experience the classical events and must infer the quantum ones. A game has the potential to allow both to be experienced. Even better, a game invites creative engagement.

After getting the idea for Quantum Tic-Tac-Toe, it took only 30 minutes to develop the rules. It felt more like a process of discovery than one of invention. Its metaphorical closeness with quantum physics continues to surprise and delight us - our hope is that it will do likewise for you.

The Rules

Quantum Tic-Tac-Toe is played on the same board as Classical Tic-Tac-Toe, and by the same two players, (X and $\mathrm{O}$ ), who take turns with $\mathrm{X}$ moving first. The start- ing position for both versions is an empty board. It is useful, however, to number the squares from 1 to 9 , left to right and top to bottom. Just for clarity, we have also chosen to double up the horizontal and vertical lines that define the Tic-Tac-Toe board when the quantum rules are in effect. When we have occasion to use the classical board, single lines will be used.

While the boards are substantially the same, the moves in Quantum Tic-Tac-Toe are different in a defined way. The differences are formally summarized below, but each rule requires a little explanation, as there is jargon unique to quantum games.

\section{The rules for Quantum Tic-Tac-Toe are the same as for Classical Tic-Tac-Toe except...}

1. Players take turns making quantum moves with $\mathrm{X}$ going first. Quantum marks are pairs of X's or O's subscripted with the number of the move. In a quantum move, two different squares are marked at once - a quantum move looks like a classical move that is half in one square and half in another.

2. Quantum moves may entangle by sharing squares. An entanglement arises when two or more quantum moves share a square.

3. On a cyclic entanglement, the other player chooses the collapse to classical moves. Indicate the kept halves, one per square, and eliminate the others. A cyclic entanglement occurs whenever there is a path from one square back to itself. In a collapse the quantum moves are replaced with classical moves.

4. First player to get 3 in a row of classical moves wins. For simultaneous 3-rows, the earlier in time gets a full point, the later a half point. An earlier in time 3-row has the lower largest subscript.

Rule 1 specifies that each player must indicate two squares as equal possibilities for the location of their move. Each move is half in one square and half in the other. We won't find out till later in the game, which square that move was actually in. This will involve a measurement process, and a collapse from quantum moves to classical moves. Every quantum move is in a mixed state, a superposition of two classical possibilities.

To indicate this, the traditional marks, X's and O's, are subscripted with the number of the move. Therefore, 
all $X$ 's end up subscripted with odd numbers $(1,3,5,7,9)$, while all O's end up subscripted with even numbers $(2,4,6,8)$. Two subscripted marks will be used to indicate a complete quantum move.

Rule 2 specifies that since the "quantum" moves of the first rule are potential, not actual, squares can hold more than one mark. Until a measurement occurs, an unlimited number of "quantum" moves may be indicated in any uncollapsed square. Such moves, which share a square, are entangled. Since only one move can end up in the shared square, where one ends up influences where the others end up. This situation, two moves into a game, is shown in Figure 1.

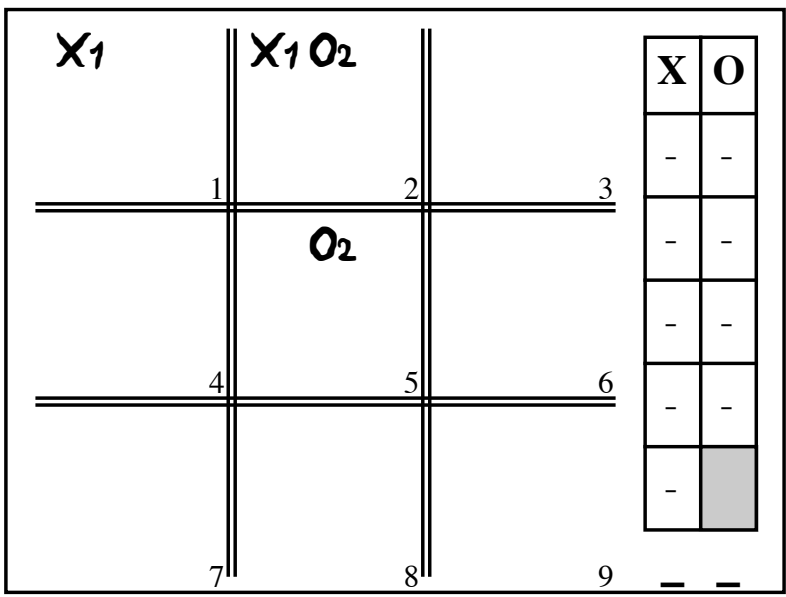

Figure 1

If Quantum Tic-Tac-Toe is to faithfully capture the essentials of quantum mechanics, then there will need to be a measurement process and a transition to the classical state.

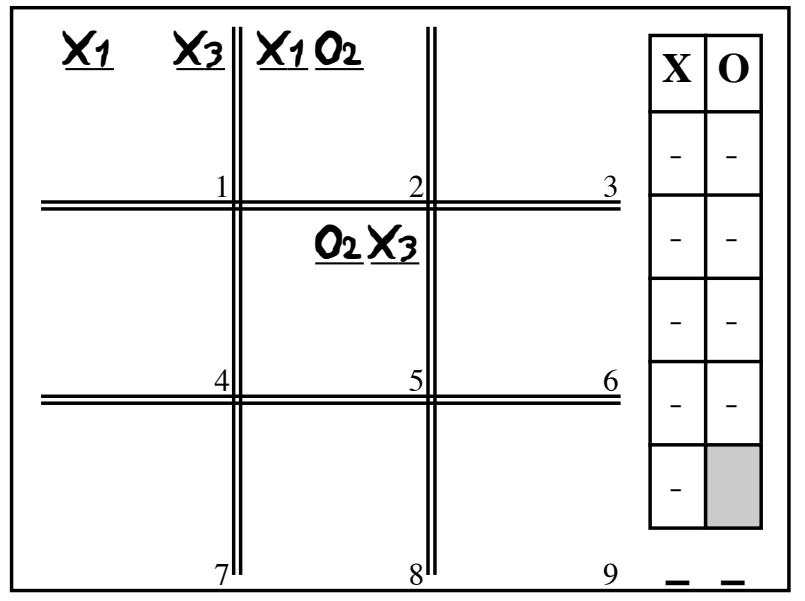

Figure 2
Rule 3 specifies the measurement mechanism. Consider Figure 2. X's next move has created a cyclic entanglement among the first three moves of the game, which have been temporarily underlined for clarity.

Starting with any half move, the link of entanglements leads back to that half move. For instance, start with move $X_{1}$ in square 1 . It depends on move $X_{3}$, which depends on move $\mathrm{O}_{2}$, which in turn depends on $\mathrm{X}_{1}$. Therefore, $\mathrm{X}_{1}$ depends on $\mathrm{X}_{1}$, an inherently selfreferential dependency.

The measurement process begins with a cyclic entanglement. It turns out, that no matter how complicated the cyclic entanglement, it only has two possible ways to collapse to classical moves. The two possibilities are shown in Figures 3 and 4, where enlarged bold markers indicate the "classical" moves, and the plain text markers indicate the "evaporated" moves. The plain text markers may be kept for historical purposes, but will play no other role in the development of the game. They have ceased to exist.

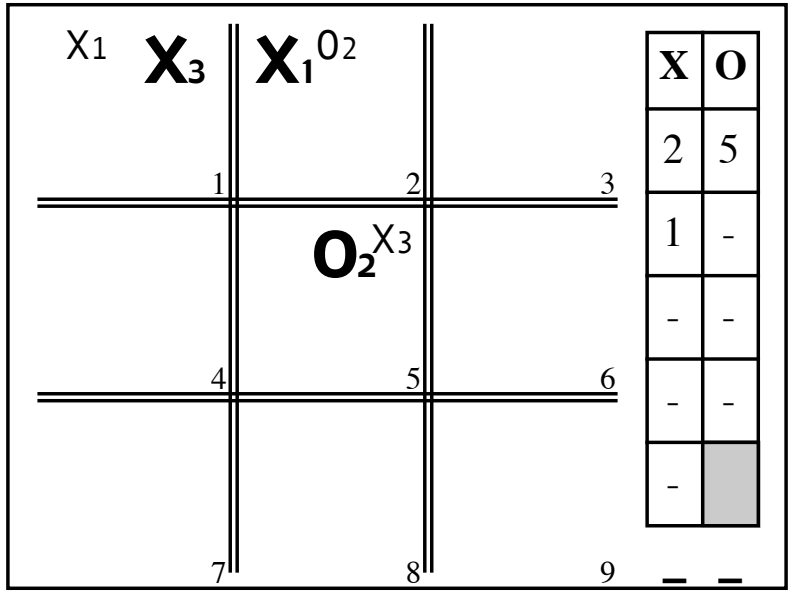

Figure 3

Since $\mathrm{X}$ made the move that caused the cyclic entanglement, it falls to his opponent to decide which way it collapses. This helps balance the power in the game between the two players. In Figure 3, $\mathrm{O}$ opts for the center square which forces $\mathrm{X}$ into squares 1 and 2 . From the classical point of view, we are now three moves into the game, which is indicated by the listing of classical moves to the right of the board.

Note that $\mathrm{O}$ has just made an entirely new type of move. She chose which way the collapse occurs. In the same way that she has to decide where to place the 
two halves of her quantum moves, she has had to decide which way to resolve the collapse. After this she still gets her regular quantum move.

Also, once a square has collapsed to a classical move, quantum moves are no longer permitted in that square.

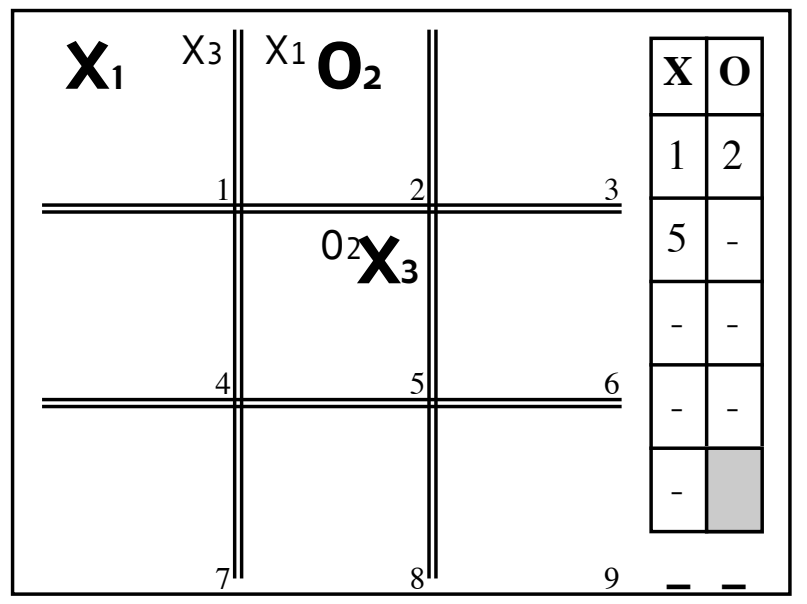

Figure 4

It is useful to have a jargon to clearly distinguish what type of move is being discussed. Therefore, Quantum Tic-Tac-Toe consists of quantum moves and collapse moves. The players take turns making quantum moves, but only get to make a collapse move if their opponent's last quantum move formed a cyclic entanglement. While there must be at least one collapse move in every game, it turns out there cannot be more than four, and how many depends critically on how play evolves. Except at the conclusion of the game, a player who makes a collapse move gets to make their next quantum move immediately afterward.

Figure 4 shows the other possible collapse. It is not as favorable for $\mathrm{O}$, so it is strategically unlikely for her to make this choice, but it is one of the possibilities. The listing of classical moves still involves the same three squares, but their sequence, and who is in them, is now different.

As a practical matter, $\mathrm{O}$ can indicate the collapse she chooses by simply marking any one of the quantum half moves in any of the squares which are a part of the cyclic entanglement. From that single choice, all the other squares are uniquely determined.

Another way to look at the criteria for collapse is to notice that there are now three moves in three squares. We can't permit any more, since four moves won't fit in only three squares. In order to avoid a potential im- possibility on the next move, it is necessary to preemptively prevent it by collapsing from the quantum states to the classical ones.

One subtlety of an entanglement is that not all of the squares involved have to reside on the cyclic part. Some can lie off of the cyclic portion as "stems". Regardless of which of the two possible ways the cycle collapses, the stems are determined by their very structure to collapse in only one way. An example of this is shown in Figure 5.

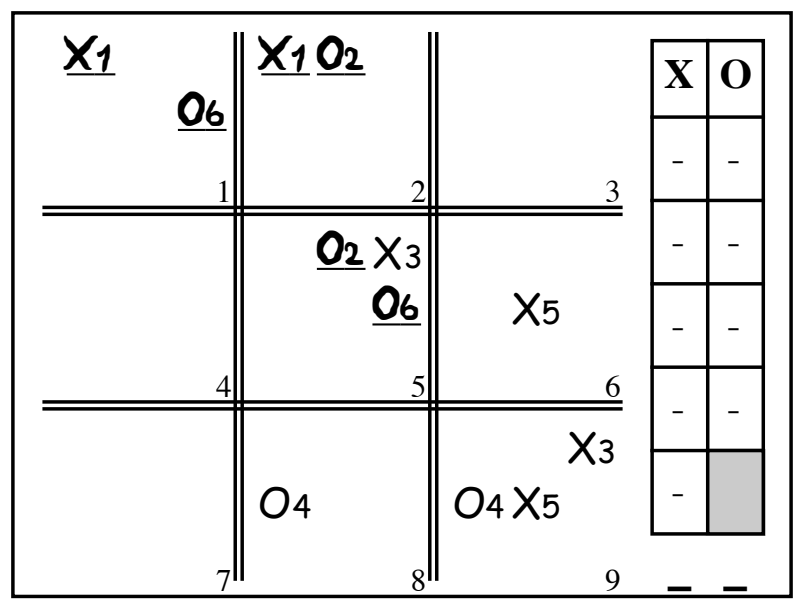

Figure 5

Moves one, two and six are on the cycle and have again been underlined for emphasis. Moves three, four and five, are on a single stem with a branch and are shown in a lighter font. Move three must collapse into square 9 because either moves two or six will end up in square 5. Similarly, moves four and five must collapse into squares 8 and 6 respectively, since move three will end up in square 9.

Any attempt to select a stem square as the square to specify a collapse in, leads either to not resolving the cyclic part, or to making its resolution impossible.

Since $\mathrm{O}$ made the move that formed the cyclic entanglement, $\mathrm{X}$ gets to choose how it collapses. If he is not careful, he can give the game to O. Figure 6 shows the resulting win for $\mathrm{O}$ if $\mathrm{X}$ specifies the collapse such that $\mathrm{X}_{1}$ ends up in square 1 .

A game of Quantum Tic-Tac-Toe is not over until at least one player gets a 3-row of classical moves. As in Classical Tic-Tac-Toe, the 3-row can be horizontal, vertical, or diagonal. 


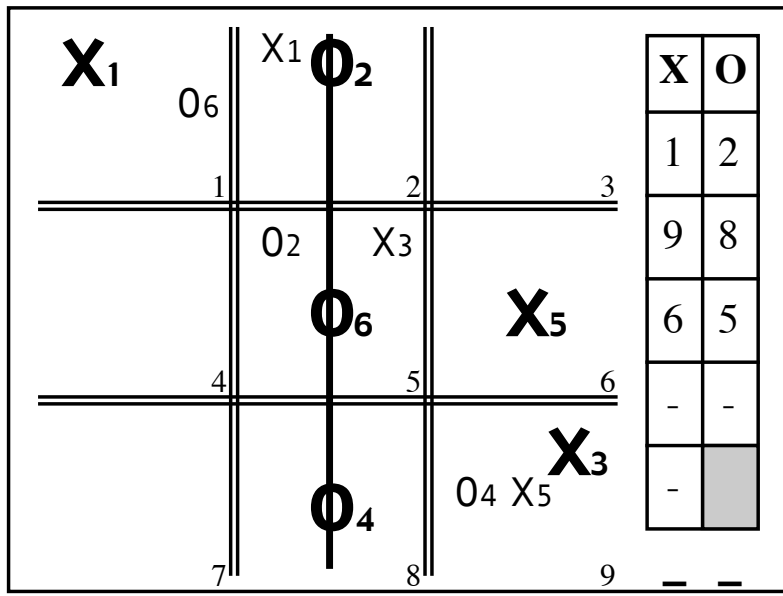

Figure 6

It is occasionally useful to list the actual quantum moves. This is shown in Figure 7 for the game played to completion in Figure 6, with the collapses circled.

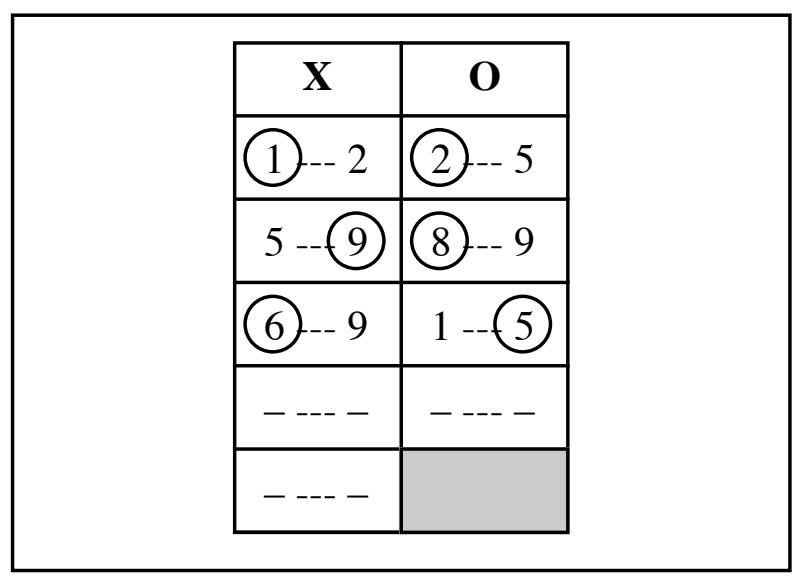

Figure 7

Both squares are indicated, but their order is not important.

In all the situations shown so far, all the moves on the board were entangled together. Figure 8 shows an example of multiple separate entanglements.

The first two moves form one entanglement, while the next three moves had formed another, which was just collapsed. Separate entanglements collapse separately. In the classical listing the second three moves are shown as their collapsed values, but the first two moves are still in their uncollapsed quantum state.

Since the classical moves only show up as the result of a collapse, they appear in groups, instead of one-at-atime as in Classical Tic-Tac-Toe. This leads to the sur- prising result that both $\mathrm{X}$ and $\mathrm{O}$ can get a 3-row at "the same time". Such a situation is shown in Figure 9, where the cyclic entanglement is shown underlined, and the stems in a lighter weight font. The isolated halves of the stems have also been centered in their squares for clarity.

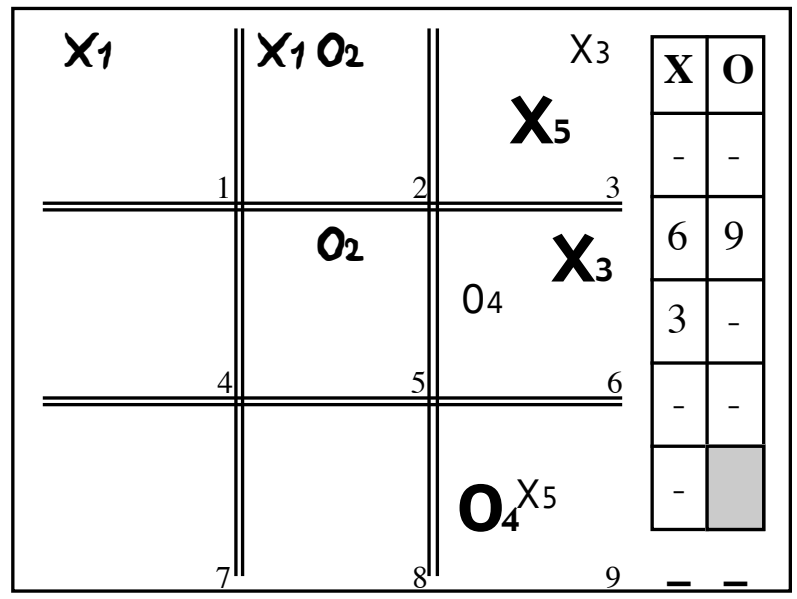

Figure 8

Player $\mathrm{X}$ has just formed a cyclic entanglement between his first and last moves, and is confident that no matter how $\mathrm{O}$ resolves the collapse, he will have a mark in square 1 and therefore a 3-row in the left column. However, either resolution gives $\mathrm{O}$ a 3-row also, in the right column. Rule 4 specifies how to score this situation, but there are two reasonable alternatives to our specification that merit discussion.

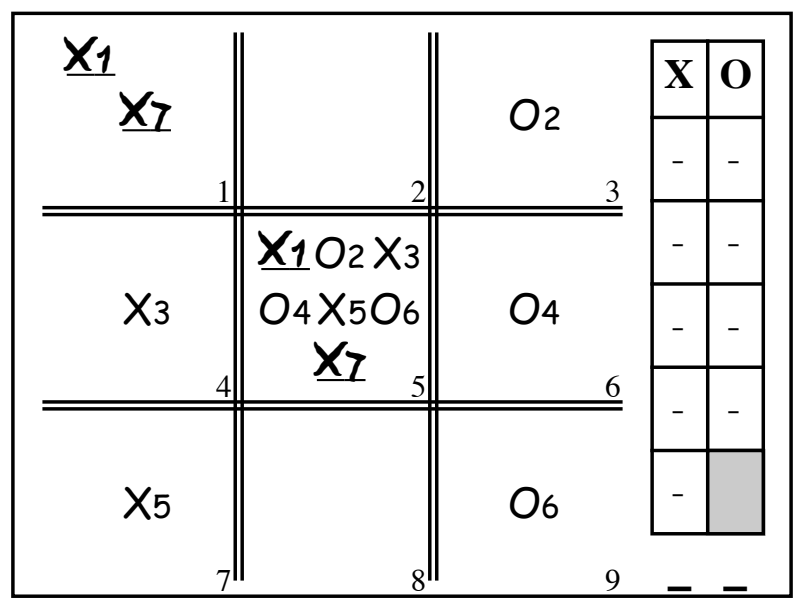

Figure 9

It could be argued that this is a tie game. The only tie game possible in Classical Tic-Tac-Toe is the "cat's game", where neither player gets a 3-row and the effective score is zero to zero. Quantum Tic-Tac-Toe would seem to support tie games with positive scores. This 
happens because as viewed from classical time, the game moves forward in unpredictable sized clumps. Several quantum moves transition to their classical values all at once.

However, it could also be argued that $\mathrm{O}$ simply wins this game. She cleverly chose the collapse such that $\mathrm{X}$ 's last move ends up in square 1 instead of square 5 . While both players get a 3-row "at once", a strict interpretation of the listing of classical moves indicates that $\mathrm{O}$ got her 3-row on move six of the game, while $\mathrm{X}$ did not get his 3-row until move seven.

We find more merit in the argument that if one player can delay the occurrence of a win by their opponent long enough to grab one of their own, then the skill or tenacity required to do so, merits some credit. If $\mathrm{O}$ had chosen the other collapse, it is $\mathrm{X}$ that would have had the earlier in time 3-row.

Therefore Rule 4 specifies a full point to the earliest in classical time 3-row, and a half point to any others that show up as a part of the final collapse of the game. The 3 -row that occurs earlier in classical time is the one with the smaller largest subscript. This situation is shown in Figure 10, where the earlier 3-row is indicated with a solid line, and the later 3-row is indicated with a dashed line. The smaller largest subscript is $\mathrm{O}_{6}$ versus $\mathrm{X}_{7}$, for the collapse $\mathrm{O}$ chose. If $\mathrm{O}$ had collapsed the cyclic entanglement the other way, it would have been $\mathrm{X}_{5}$ versus $\mathrm{O}_{6}$ and $\mathrm{X}$ would have won the game.

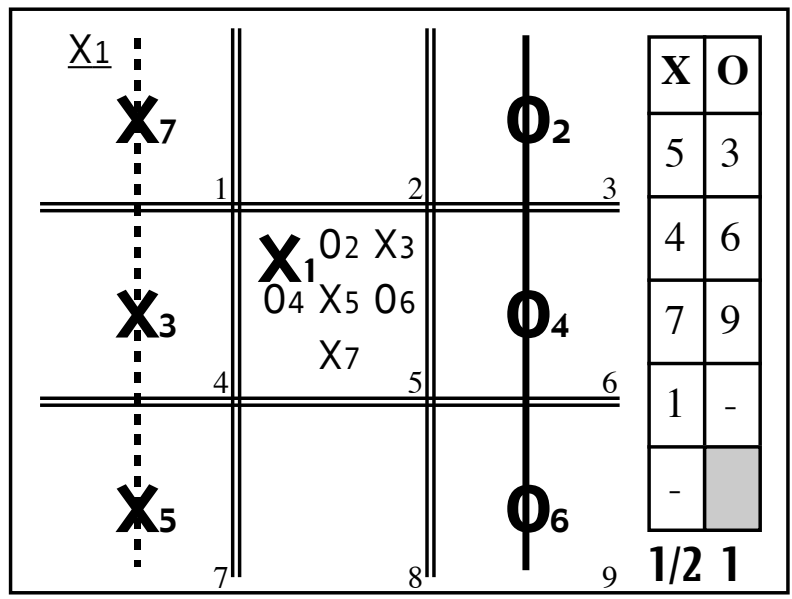

Figure 10

There are a few games, where if $\mathrm{O}$ plays very badly, $\mathrm{X}$ can actually get two 3-rows for himself. If they share $\mathrm{X}_{9}$, $\mathrm{X}$ gets 2 points, if they don't $\mathrm{X}$ gets $1-1 / 2$ points.
A final special rule is assumed, since it is forced upon us by the boundary condition of an all-but-full board at the end of the game. Normally, all quantum moves must specify two squares. However, if only one open square remains, all of the other eight having collapsed to classical states, then $\mathrm{X}$ is permitted to place both halves of his last quantum move into the remaining playable square. This situation is shown in Figure 11.

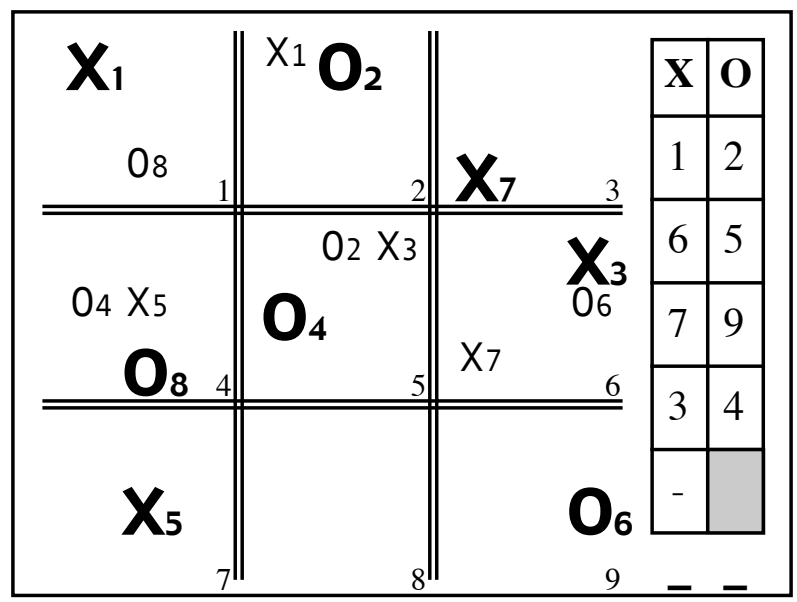

Figure 11

At the end of move eight, the board contains a single entanglement, whose cyclic part traverses squares 1,2 , 5 , and 4, with two stems, one off square 4, and another off square 5 with a branch to squares 3 and 9. O's last move formed the cyclic entanglement, which $\mathrm{X}$ chose so as to avoid giving $\mathrm{O}$ a win down the main diagonal. Once he does, there remains only square 8 as a legal move, so X may place both halves of his last move here, and we regard them as self-collapsing directly to the classical state without any choice possible.

\section{The Mapping to Quantum Physics}

In this section we apply the game of Quantum Tic-TacToe as a metaphor for quantum physics. Parts of this metaphor are built in, designed into the rules; other parts are emergent, these parts are consequences of the rules. To the extent that the emergent metaphors are deep and extensive, we can have confidence that the rules capture, in some essential sense, the essence of what quantum physics "means".

The built-in metaphors come from Rules 1 and 2. They are the mixed state nature of the quantum moves, and the fact that they can be entangled with other quantum moves. Rule 3 is subtly implied by the first two rules, so the measurement mechanism is actually an emergent metaphor. Rule 4 leads to no metaphorical elements since it only deals with points for winning. All the 
other metaphorical elements of Quantum Tic-Tac-Toe are emergent. To see this, an alternative frame of reference needs to be established.

The rules of a game specify the legal states the game can be in, and the legal transitions from one state to another. However, it is also possible to regard the rules of a quantum game as meta-rules on the underlying classical game. When a move is made in Quantum TicTac-Toe, it can be interpreted as indicating the existence of multiple games of Classical Tic-Tac-Toe in simultaneous play. If $\mathrm{X}$ marks two squares, and then $\mathrm{O}$ marks two more squares, the quantum game is a metalevel view of four simultaneous classical games. This is shown in Figure 12.

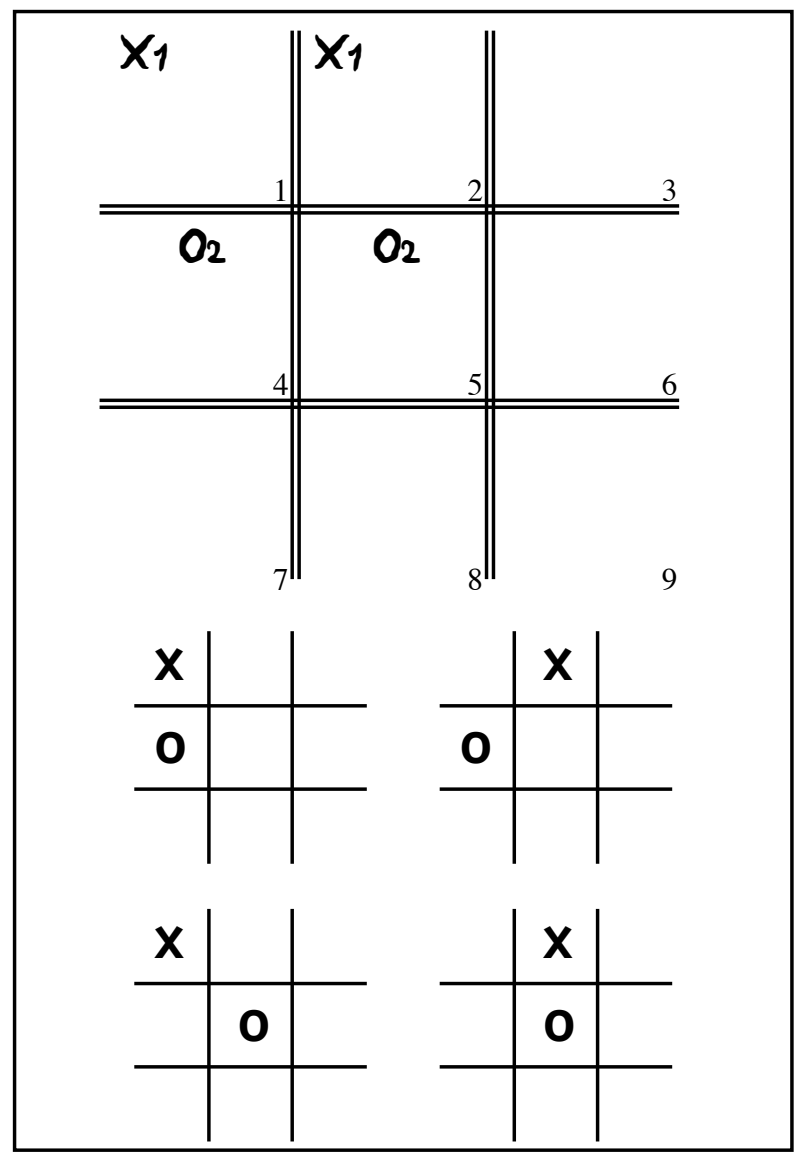

Figure 12

Each additional quantum move that doesn't entangle with any preexisting moves doubles the number of classical games in play. For a quantum move that entangles with a preexisting entanglement on only one square the increase in the number of classical games is the product of the permutations of the other entanglements on the board. This can range from 1 to 12 .
In general, the number of simultaneous classical games, $N_{c}$, is equal to the product of the permutations of the various entanglements, $n_{e}$;

$$
N_{c}=\prod_{1}^{n_{e}} P_{e}
$$

where the number of permutations, $P_{e}$, is just one more than the number of moves involved in the entanglement, $n_{m}$;

$$
P_{e}=n_{m}+1
$$

The doubling one might expect is thwarted by the contradictory games that are implied by straight permutations. This is shown in Figure 13.

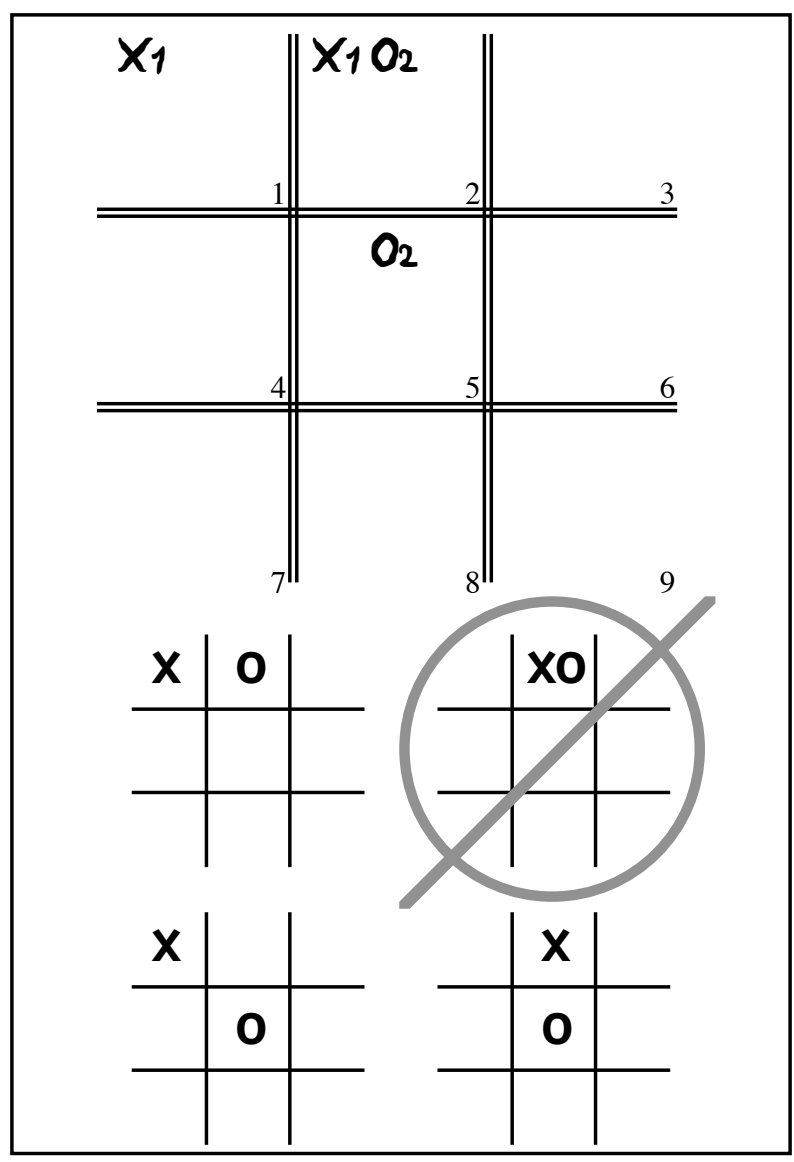

Figure 13

From now on the contradictory games will be suppressed. It is interesting to watch what happens to the number of classical games in play as an entanglement grows toward eventual collapse. Figure 14 shows one more move past Figure 13. 
indeterminate because it is up to the choice of the player which classical game gets continued. While O's entanglement eliminated games b and c, $X$ still needs to choose whether to eliminate a or $\mathrm{d}$.

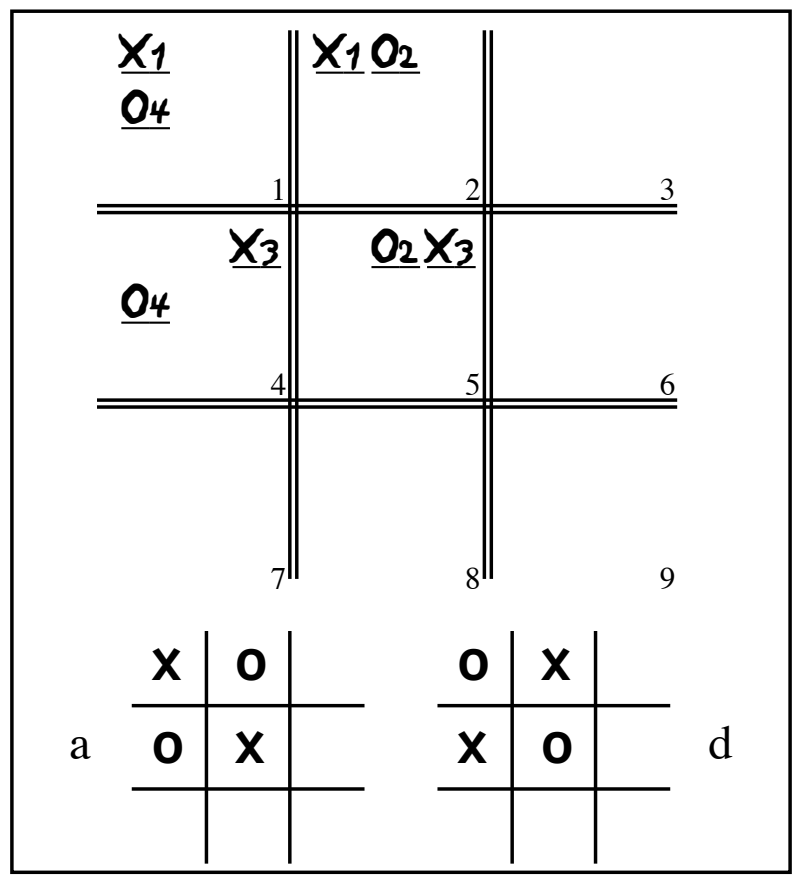

Figure 15

If collapse of the wave function in quantum physics, also has this two-stage nature, then it is less of a wonder why the measurement problem has gone unsolved for so long. What had been thought of as being a single event may actually be two interconnected events, each masking the nature of the other.

We are now ready to consider what emergent metaphorical elements Quantum Tic-Tac-Toe can provide about quantum reality.

The first emergent metaphorical element is the existence of measurement events. A measurement event is driven by a specific deterministic process, cyclic entanglement, and concludes with a nondeterministic transition to a classical state. The transition reduces (collapses) the degree of indeterminacy inherent in the superposition of classical games.

In addition, we have two different mechanisms for changing the state of the system. The first is the making of quantum moves, and the second is the making of collapse moves. Let's liken the first to the evolution of the wave function, and the second to the collapse of the 
wave function. This mimics the evolution/collapse duality of quantum mechanics.

Furthermore, it is not hard to generalize the idea of quantum games so that this duality mimics even better the pattern in quantum physics, where the evolution of the wave function is deterministic, but the collapse is not. In Quantum Tic-Tac-Toe both are. However, TicTac-Toe is an extremely simple game, and if the quantum moves were to be predetermined, there would be little left to play with. A more challenging game could easily have the quantum moves proceed deterministically, pausing whenever a cyclic entanglement occurred to allow the players to make choices as to how they collapsed. This establishes a determinism/nondeterminism duality as well.

As the game proceeds, collapses tend to happen, and the board fills up with classical moves. In the limit, Quantum Tic-Tac-Toe begins to look like Classical TicTac-Toe. This would be even more apparent on a board with many squares where new moves tended not to stray too far from recent moves. In physics, the tendency for large quantum systems to behave indistinguishably from classical ones is called the correspondence principle.

However, the way the quantum system approaches the classical limit is to do so in clumps of moves all-atonce, and not necessarily in order. This metaphor speaks to the fuzziness of time in the present.

Consider the Quantum Tic-Tac-Toe game shown in Figure 16. On move one, $\mathrm{X}$ has staked out the center and a corner, a good strategy based on what works in Classical Tic-Tac-Toe. O does similarly taking advantage of the fact that $\mathrm{X}$ is not yet deterministically in control of the center square, and at the same time preventing $X$ from ever getting a 3-row down the antidiagonal. Moves three and four proceed for similar tactical reasons. On move five $\mathrm{X}$ makes a very threatening play that $\mathrm{O}$ must respond to. While her decision to create a cyclic entanglement gives selection of the collapse to $\mathrm{X}$, any other move allows $\mathrm{X}$ to force a win. $X$ chooses to collapse the entanglement such that he ends up with a single horizontal 2-row, even though this gives $\mathrm{O}$ two 2-rows. $\mathrm{X}$ can force a win, 1 to $1 / 2$ from here by playing in squares 4 and 8 .

However, the classical listing of moves at the end of move six, just after the collapse, makes no tactical sense at all. O could have easily won the classical game on move six simply by playing in square 4 , and $\mathrm{X}$ should have played there previously on move five to prevent it, but neither $\mathrm{X}$ nor $\mathrm{O}$ could force these outcomes in the Quantum game. These are not the only poor moves in the classical version. O should have played her first move in the center, and when she didn't, X should have played his second move in either remaining corner. Of the first six moves in the implied classical game, four of them were tactically dumb.

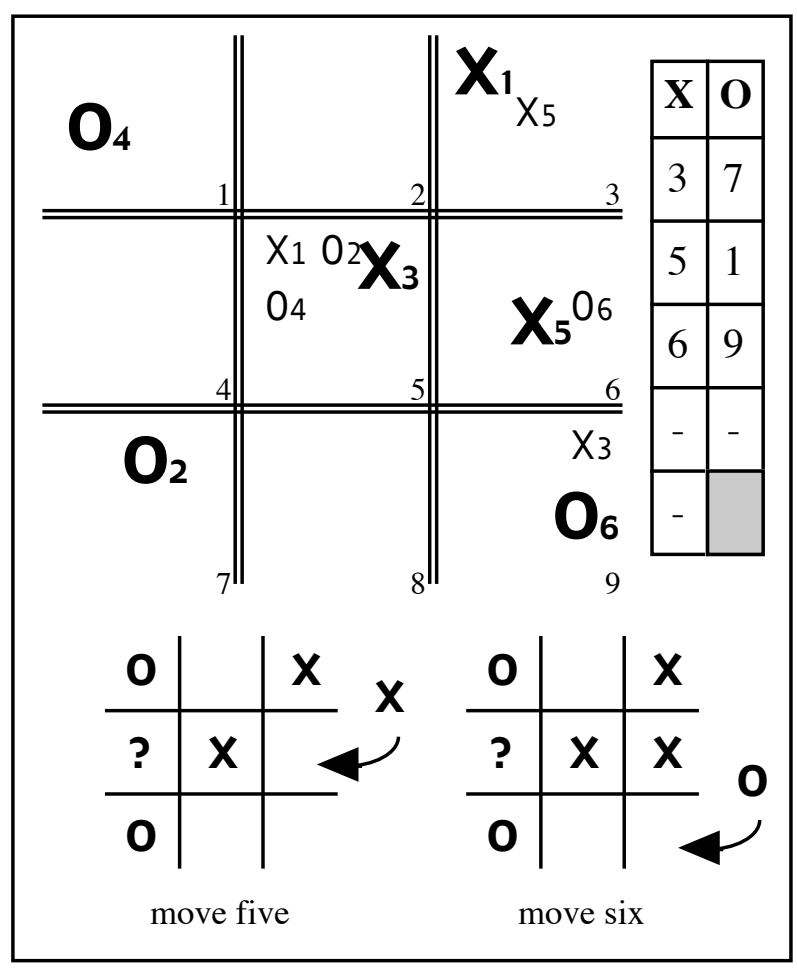

Figure 16

If all an observer has access to is the classical listing, this is a very mysterious result. If the observer is persuaded that $\mathrm{X}$ and $\mathrm{O}$ are both competent Tic-Tac-Toe players intent on winning, he must conclude that the classical game he witnessed was actually played under quantum rules. Thus it is possible in general, just from the classical listings, to ascertain whether a game of Tic-Tac-Toe was played under quantum or classical rules.

Although we usually take it for granted, it is actually a little remarkable that we have been able to deduce that we live in a quantum universe and not a classical one. As it is, this revelation is less than 100 years old. Call this the ascertainity principle.

We believe this next metaphor is an unrecognized one. Let's return to the game shown in Figure 10, and ask two questions about cause and effect. Consider the very first move of the game. Our first question is, 
"What caused move one to be half in square 1 and half in square 5?" Easy enough - it was the first quantum move of the game, an immediate effect generated by a preceding cause. Our second question is, "What caused move one to be in square 5?" More interesting - move seven did, a later move "caused" an earlier move! This is an example of backwards in time causality. It is perfectly benign in Quantum Tic-Tac-Toe, and probably in most quantum games as well. If we are ever to defeat the speed of light, the universe will have to support backwards causality. There is hope therefore, that this is not as serious an objection against the possibility of breaking the light barrier as has been previously assumed.

Such a specific and benign backwards causality mechanism is perfectly adequate to deal with the Delayed Choice experiment ${ }^{3}$. Until the photon is detected, two universes, one where it went one way around the lens galaxy, and one where it went around the other way, are still both in play.

Let's segue to the concept of a game tree. A game tree is simply a graph of all possible state transitions. It might be a directed graph or a pure tree; it depends on the rules of the game under consideration. Classical Tic-Tac-Toe is a pure tree if subscripts and numbered squares are used, a substantially smaller directed graph if they are not. The game tree for Quantum Tic-TacToe is a pure tree.

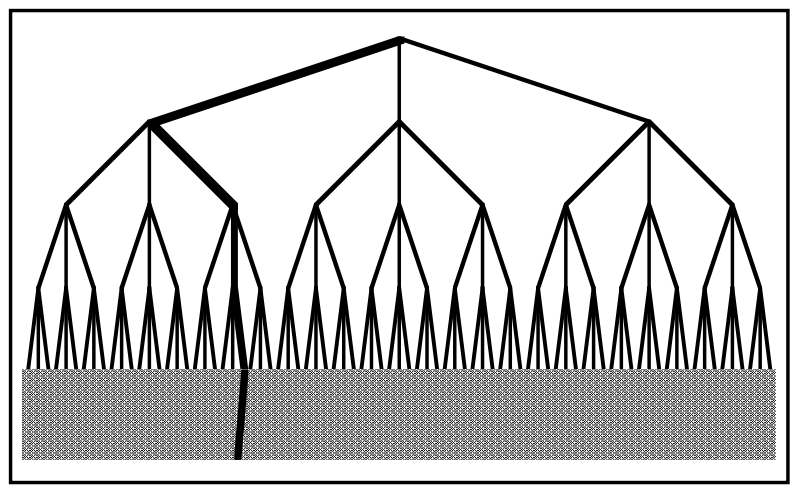

Figure 17

Figure 17 is a schematic of a particular instance of a classical game. It is represented as a single path from the root (the starting position) to whatever ending node was reached. While a quantum game has its own (much larger) game tree, and any instance can likewise be represented as a single path down that large tree, when regarded as meta-rules for the underlying classical game, it is possible to map an instance of a quantum game onto the classical game tree. Only now, an instance of a quantum game is a many-branched path, which tends to grow wider with each quantum move and narrower with each collapse move. Figure 18 shows the quantum multi-branched tree with a collapse on move four to the same classical game as shown in Figure 17.

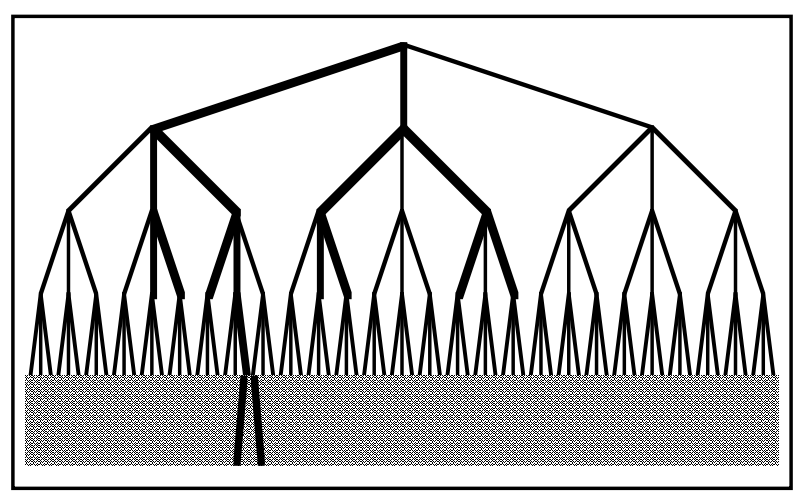

Figure 18

This view explains the allure of Everett's Many Worlds Hypothesis ${ }^{4}$. There are indeed many classical realities in simultaneous existence, but in this view their number does not increase indefinitely with each successive quantum event. While the evolution of the wave function expands the tree, each measurement thins it.

Because trees tend to grow exponentially, Quantum Tic-Tac-Toe also demonstrates the exponential processing promise of quantum computing. Not counting symmetries, the total number of possible games of Classical Tic-Tac-Toe is 9!. For Quantum Tic-TacToe, the total number of possible games is $9 !^{2}$. The computational cost of programming a computer to play Quantum Tic-Tac-Toe is exponentially larger than for Classical Tic-Tac-Toe. A quantum computer reverses this ratio, calculating simultaneously in the superposition of states an answer to be delivered to one final classical instance.

One of the mysteries of quantum physics is the sumover-histories calculation. How can the possible paths not taken by a particle have had an impact on the path it does indeed take? There is no mystery here for games. The effect of possible futures on the present is precisely the concern of strategy. The actuality that occurs has always been strongly influenced by futures that never happened.

Quantum games simply extend this into the past. The actualities that occur are strongly influenced by pasts 
that never happened. Consider the strategic situation depicted in Figure 19.

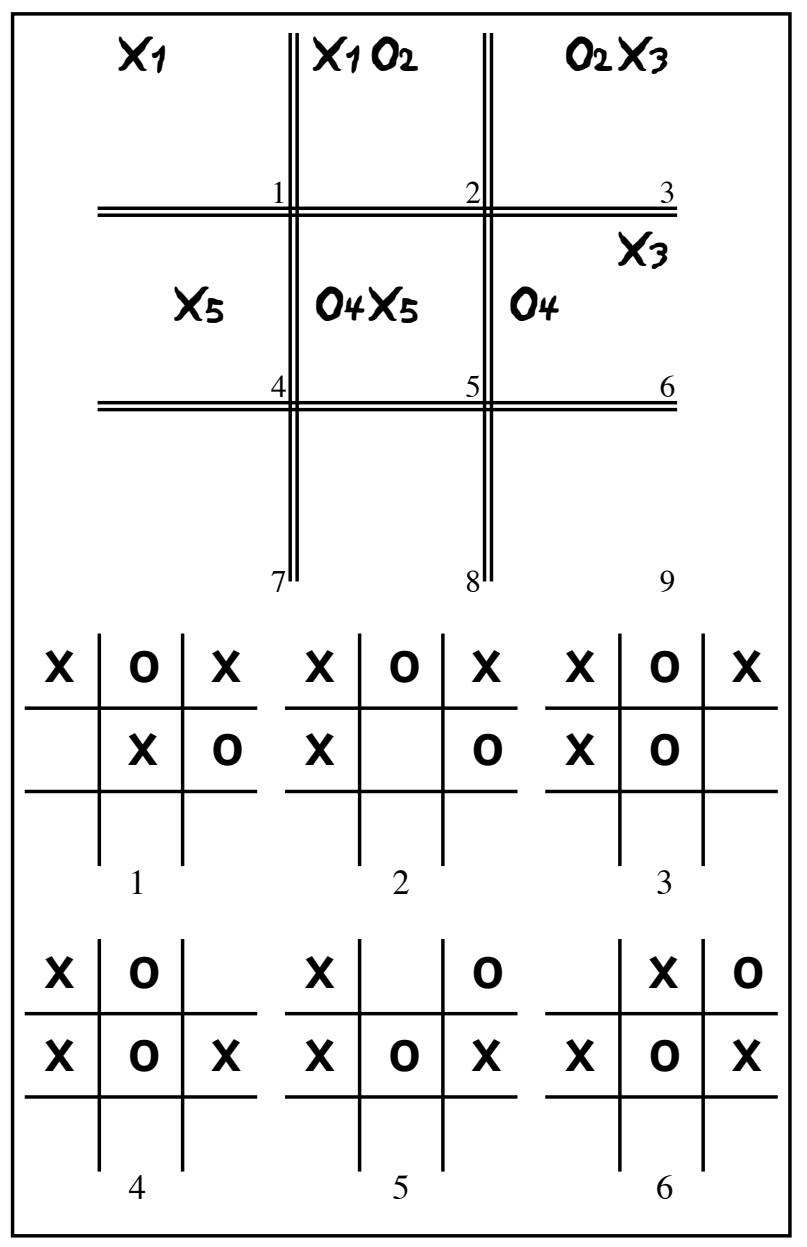

Figure 19

The five moves in this game form a single entanglement covering six squares so there are precisely six classical games in play. If $\mathrm{O}$ were to choose to create a cyclic entanglement, these six games would become two. She can in fact choose which two out of these six continue, simply by playing in the appropriate two squares. Let's number the classical games 1 to 6 , left to right and top to bottom, so they are easier to refer to. $\mathrm{O}$ wants to avoid the first two games, as $\mathrm{X}$ has two 2-rows in the first game, and one in the second, and $\mathrm{O}$ has none. In the next three games, both have a single 2row, although in game 5 converting them to 3 -rows involves the same square for both players. Only game 6 offers $\mathrm{O}$ a 2-row without giving one to $\mathrm{X}$, so $\mathrm{O}$ might reasonably choose as her strategy to move such that classical games 5 and 6 continue. $\mathrm{X}$ will have to select which one of them the entanglement collapses to.
To do so, O merely makes a quantum move with each half in those two squares of the entangled squares which are empty in the classical games she wishes to keep. Therefore, $\mathrm{O}$ plays $\mathrm{O}_{6}$ in squares 1 and 2. $\mathrm{X}$ does not want game 6 , which now offers $\mathrm{O}$ two 2-rows, so he selects the collapse where $X_{1}$ is in square 1 , yielding game 5 with $\mathrm{O}$ in square 2. $\mathrm{X}$ now has a 2-row, while $\mathrm{O}$ has two, where one of them requires the same square (7) for completion as X's. We leave as an exercise for the reader to show that while $\mathrm{O}$ cannot prevent $\mathrm{X}$ from winning, she can at least earn a half point for herself.

It should be clear from the above analysis that the games in existence at the time move six was made had a major impact on the choice of that move. Similarly, both games that were left after the cyclic entanglement influenced the choice of the collapse. All this, even though when the quantum game is finally over, these classical games are gone with no record of their existence. It is hard to avoid the philosophical issues. Were these classical games real? Did they ever exist? If they were/are a reality, where are they now? They certainly had an impact on the potentiality that ended up becoming the actuality.

As we struggle to express these ideas, it seems that our language is hardly adequate to the task.

Perhaps what bothered Einstein most about quantum physics, was the "spooky action at a distance" implied by entangled states. Quantum Tic-Tac-Toe suggests an explanation. The "action" is spooky because it occurs between universes - the situation in one classical instance influences the outcome in another. As long as our experiments constrain us to classical events, there are no possible mechanistic explanations. The classical model does not support cross-universe cause and effect connections, but it seems that the quantum model does. When one universe ceases to exist, its effects are immediate, unmitigated, and undiminished.

The game metaphor implies that the simultaneous classical games are "universes" implied by the quantum state. While there is no speed-of-light limit in Quantum Tic-Tac-Toe, it at least suggests spooky action at a distance.

The relationship of stems to the cyclic entanglements they connect to (Figure 5) also offers insight into quantum physics. Consider a photon impinging on a polarization detector. If cyclic entanglement of quantum states is the measurement mechanism, where is this entanglement for the photon? The cyclic entanglement occurs within the large ensemble of atoms in the de- 
tector, the photon is just a stem off this cyclic structure. In general, then, a macro measurement of a quantum object consists of a cyclic entanglement within the macro object, which the quantum object has become entangled with, but which is not itself a part of the cycle. When the detector collapses, which it does over and over again due to thermal vibrations, with or without a photon involved, the photon is taken down with it.

One of the prototypical experiments to demonstrate the counter intuitive nature of quantum physics is the double slit experiment. If we do not observe which slit the electron goes through, we are compelled by the pattern of experimental results to conclude that the electron went through both slits, even though we have never observed an electron as other than an undivided whole.

Consider Figure 20, where the game is over, but X's first move never collapsed.

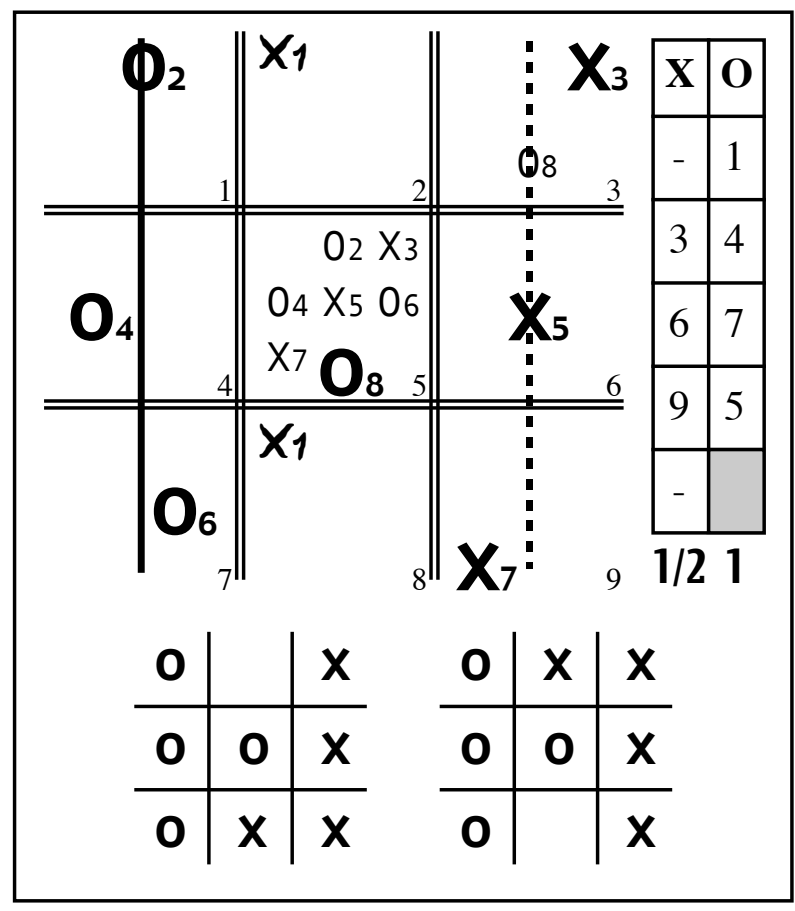

Figure 20

In this case, two classical games are left active at the conclusion of the quantum game. While the possibilities associated with $X_{1}$ influenced the course of the game, when all was said and done, move one remained in both squares ( 2 and 8 ). Having both slits open influences where the electron is eventually found, but as long as it is not collapsed at the slits, it goes through both. The historical structure of its "trajectory" included two universes. Similarly, Quantum Tic-Tac-Toe supports uncollapsed historical states. The game tree is multi-branched all the way to its conclusion.

We leave as an exercise for the Reader the proof that 27 is the maximum number of simultaneous games of Classical Tic-Tac-Toe, and that 9 is the maximum simultaneous wins achievable by either $\mathrm{X}$ or $\mathrm{O}$. Note that such games are unlikely, given competent players with an intent to win, but they are legal games.

While every cyclic entanglement has precisely two possible collapses, whenever a cyclic entanglement consists of only X's or of only O's, there is only one possible classical position it can collapse to. Since moves in Classical Tic-Tac-Toe are not subscripted, one X (or O) on the board is formally indistinguishable from another. In quantum physics, indistinguishable particles interact with a different statistical distribution than distinguishable particles. Therefore, Quantum Tic-Tac-Toe also exhibits indistinguishable object statistics. Since the statistics of indistinguishable particles plays a central role in our FTL protocol, it was gratifying to see this metaphor show up as well.

Randomness remains a challenging concept to formalize precisely. Any mechanistic definition ends up being equivalent to a pseudo random number generator. Given the parameters to the pseudo random number generator, the sequence is perfectly deterministic and predictable. We believe that the strongest formalization of randomness derives from formal game theory ${ }^{5}$ and the tension in competitive situations.

A good example of this is the decision game "Paper, Rock, Scissors". Two players simultaneously reveal their move, a choice of paper, rock, or scissors. The rules are that paper covers rock, rock breaks scissors, scissors cut paper, with ties redone. The strategy in this game is to avoid revealing any pattern in one's choices that an opponent could take advantage of. One is led by the nature of the game to play perfectly randomly!

While random play in Quantum Tic-Tac-Toe is not particularly effective, games in general are the premier venue where a workable definition of randomness arises in a natural fashion. Therefore, the game metaphor for reality may well be superior to the machine metaphor when it comes to explaining quantum randomness.

One of the remarkable facts about our universe is that it started in a very ordered state, with exceptionally low entropy. Penrose ${ }^{6}$ places the odds against the Universe's "starting position" at 1 in $10^{\wedge} 10^{123}$ ! The open- 
ing position of both versions of Tic-Tac-Toe is an empty board: a very ordered (low entropy) state. This is in fact typical of games in general. Therefore, the game metaphor also is consonant with the low entropy initial conditions found in our own reality.

Similar arguments apply to the fine-tuning of the universal constants ${ }^{7}$. The rules of a game are carefully chosen to lead to interesting play. They are not chosen randomly. Surprising slight variations in the values of any of the fundamental constants ${ }^{8}$ would lead to a universe inhospitable to life as we know it.

From the two built-in metaphors of Quantum Tic-TacToe we have been able to identify more than 20 emergent metaphors for quantum physics (summarized in Table 1), either directly from Quantum Tic-Tac-Toe, or indirectly from games in general, and quantum games in particular. This is suggestive that there is something fundamental about looking at reality as if it were a quantum game.

\begin{tabular}{|l|}
\hline QT3 Emergent Metaphors w/ Quantum Physics \\
\hline Measurement events \\
\hline Cyclic entanglements \\
\hline Transition to classical states \\
\hline Reduction in the number of classical "universes" \\
\hline Superposition \\
\hline Evolution/collapse duality \\
\hline Determinism/non-determinism duality \\
\hline Correspondence principle \\
\hline Fuzziness of time in the present \\
\hline Ascertainity principle \\
\hline Backwards in time causality \\
\hline Delayed Choice experiment \\
\hline Everett's Many Worlds Hypothesis \\
\hline Quantum computing \\
\hline Present influenced by futures that never happen \\
\hline Present influenced by pasts that never happened \\
\hline Spooky action at a distance \\
\hline Macro measurement of a quantum object \\
\hline Uncollapsed historical states \\
\hline Indistinguishable object statistics \\
\hline Quantum randomness \\
\hline Low entropy initial conditions \\
\hline Fine-tuning of the universal constants \\
\hline
\end{tabular}

Table 1

\section{Variations}

One simple variation on Quantum Tic-Tac-Toe is to change Rule 1 to specify three squares per quantum move. This version is substantially harder to play $\left(\square 9 !^{3}\right)$, and the permutations of classical games grow exponentially as each entanglement grows, as well as when the number of entanglements increases.

For these variations it is not clear if simple selfreference is enough to force collapse to classical moves. A stronger concept, exhaustion, may be required. Exhaustion is when the number of marks in an entanglement is equal to the number of squares involved. In 2way Quantum Tic-Tac-Toe, self-reference and exhaustion are degenerate. Determining whether collapse in quantum physics is driven by simple self-reference or by exhaustion, awaits a more mature development of the mathematics of self-reference of quantum systems.

In formal game theory, one of the major concerns is determining perfect play: can either player force a win, and how do they do it. In Classical Tic-Tac-Toe, perfect play results in a cat's game, and neither player can force a win. It is not known yet what perfect play is in 2-way Quantum Tic-Tac-Toe, but it is known what perfect play is in 9-way Quantum Tic-Tac-Toe, assuming exhaustion is the collapse method.

In 9-way Quantum Tic-Tac-Toe, each player must indicate 9 squares for their quantum move. Each move is $1 / 9^{\text {th }}$ in each square. Since there are only 9 squares on the board, each player, on every move, must place a mark in every square on the board. No collapse by exhaustion is possible until the very last move, which is made by $\mathrm{X}$, so $\mathrm{O}$ gets to choose how it collapses. At this point there are 9 ! classical games in simultaneous play, the entire game tree of Classical Tic-Tac-Toe. This is truly Everett's Many Worlds situation.

O now finds herself in the enviable position of being able to choose from the entire universe of possible Classical Tic-Tac-Toe games. This universe of possibilities is not large, only 362,880 games (assuming indices on the marks), but among them is every single instance of a classical game where $\mathrm{O}$ wins. Assuming no indices, there are 48 of them. O simply picks any instance of a classical game where she wins! It is as if she alone collapses the wave function of the universe.

In 9-way Quantum Tic-Tac-Toe perfect play grants $\mathrm{O}$ a win, every time. Indeed, for all practical purposes, she is the only player with a move; in a one move game. The quantum moves have all been reduced to deterministic status. Only the collapse move is nondeterministic. This suggests that among some of the variations between 1-way and 9-way Tic-Tac-Toe, one or the other of $\mathrm{X}$ and $\mathrm{O}$ finally gets the better of their opponent and is able to force a win. 


\section{Future Research}

One metaphor that does not emerge from Quantum TicTac-Toe is conjugate variables. Their absence, which cannot be eliminated in quantum mechanics, permits a greater clarity on the other concepts. Given the success with Quantum Tic-Tac-Toe, a logical next step is to create other quantum games, and actively seek or design one that will display conjugate variables.

Since most classical games utilize a playing board with fixed squares (let's call these Newtonian boards), it is an interesting question what kind of game, quantum or otherwise, would be necessary to produce relativistic metaphors.

A web version of Quantum Tic-Tac-Toe is available at www.ParadigmPuzzles.com, or through Novatia's web site, www.NovatiaInc.com. A portable version is available for the Palm Pilot, and a paper version on 3M Postit Notes ${ }^{\mathrm{TM}}$.

\section{SPOOKY-COINS \& MAGIC-ENVELOPES}

This section extends a metaphor first put forward by Nick Herbert ${ }^{9}$. We have broadened it to fully characterize the EPR experiment ${ }^{10}$, Bell's Theorem ${ }^{11}$, and our faster-than-light protocols using quantum nonlocality.

\section{The National Letter Service}

Since its inception the Realm has been faithfully served by the National Letter Service (NLS). All letters are delivered in special security envelopes, which prevent the contents from being observed in transit or read before the envelope is opened. The NLS may be a bit bureaucratic, but it is very precise: successive letters between any two addresses are always delivered in the same order in which they were mailed.

There are three types of envelopes, red, white, and blue, from longest to shortest. Most citizens use white envelopes, but the long ones (red) arrive sooner, while the short ones (blue) arrive later. The sum of the lengths of red and blue envelopes is equal to twice the length of the white ones.

In recent eons, the speed with which letters are delivered has come to be perceived as slow, even though nothing else in the Realm moves any faster. As the Realm has expanded, the time taken to mail letters from one end to the other has become unacceptably long.

\section{The Lovers and Merlin}

Roberto and Sue Ellen are two widely separated lovers frustrated by the slow communications forced upon them by the National Letter Service. They have contracted with Merlin, wizard extraordinaire, to devise a scheme where they may communicate faster than letter (FTL). He has agreed to try.

The scheme that Merlin comes up with, however, is not anything like what Roberto and Sue Ellen are expecting.

Since Merlin's scheme is so radical, he begins with a series of trial runs that demonstrate how the various parts of the scheme function. In the first trial run he sends a single stream of plain white envelopes to both Roberto and Sue Ellen. Each envelope contains a single coin of the Realm. When Roberto or Sue Ellen opens an envelope either a gold or a silver coin is revealed. Merlin instructs them both to keep a detailed list of the coins they receive, and lo and behold, their lists are completely random. Merlin now asks each of them to mail a copy of their list to the other. It turns out that the two lists are absolutely anti-correlated. Whenever Roberto received a gold coin, Sue Ellen received a silver one, and vice versa. After a moment's reflection, however, neither of our lonely lovers are surprised by this pattern, for they realize that all Merlin had to do was mail envelopes in pairs, and place opposite coins into each envelope in a random way.

In trial run two, Merlin still mails a single stream of envelope pairs to each lover, but now he uses spookycoins and magic-envelopes. Before placing two coins into each pair of white envelopes, he rubs them together and utters an incantation, "quanti entangliai instantainia", and informs Roberto and Sue Ellen that now how they open their envelopes will affect what kind of coin they receive. The two things they may do to their envelopes are to rotate them or flip them. If they open them without rotating them, either a gold or a silver coin is revealed. See Figure 21.

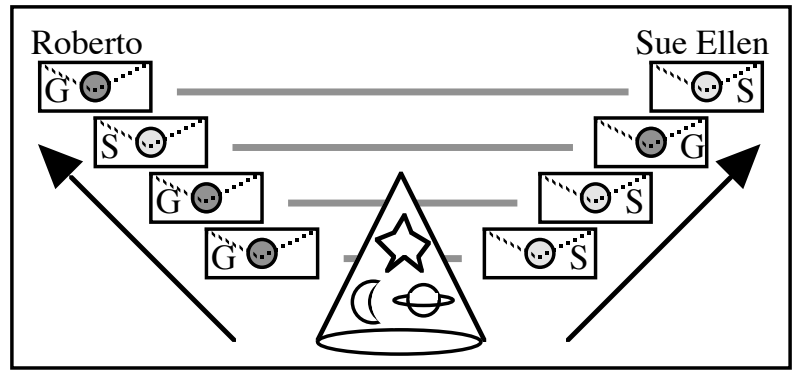

Figure 21 
If they rotate them first, then either a platinum or osmium coin is revealed. See Figure 22. If they flip them, then either a silver or gold coin is revealed; if they both rotate and flip, then an osmium or platinum coin is revealed. For any orientation of the envelope, the sequence of revealed coins is absolutely random.

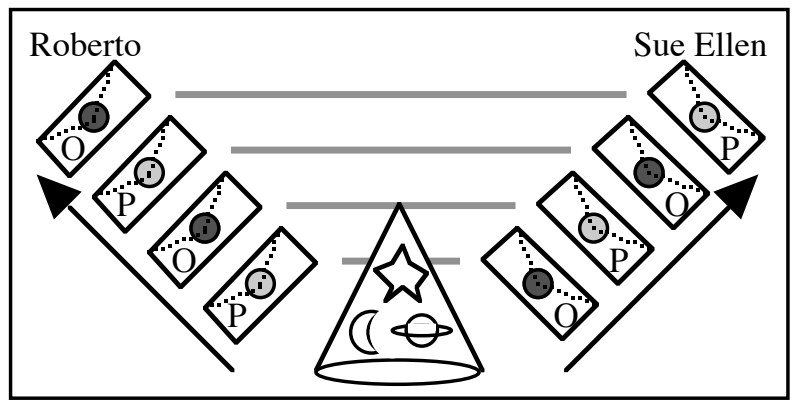

Figure 22

As before, Merlin instructs them to keep a detailed list, but now it must also record the envelope orientation as well as the type of coin they received. When they compare lists, they see four different patterns. For the case of same rotations they will observe anti-correlated lists, unless one of them flipped their envelope, in which case they see correlated lists. If their rotations are different, regardless of flip, there are no correlations at all.

Now at last, how one mail recipient opens their mail has an impact on what the other receives, but alas, because of the absolutely random pattern in the received coins, they cannot observe the difference. Roberto and Sue Ellen still cannot send information faster than letter, but they are at least getting rich in precious coins.

\section{The Sorcerer's Apprentice}

By this time Roberto and Sue Ellen are beginning to lose hope, and Merlin takes time off for a sabbatical to study nonlinear logics. In his absence, his apprentice, who understands magic-envelopes, but not spookycoins, tries to step in. Like Merlin, he places coins into pairs of magic-envelopes, but since he doesn't know how to create spooky-coins, he randomly places pairs of either gold/silver or platinum/osmium coins into them. He reasons that, since the type of coin revealed is determined by the way the envelopes are opened, that by just starting the coins out as opposites, he can do as well without spooky-coins. The apprentice's attempt is shown in Figure 23.

Since the lovers cannot see what type of coin is actually in an envelope without opening it, and since how they open a magic-envelope can change the type of coin revealed he is certain that if he is only clever enough in how he chooses to combine pairs of coins, that he can simulate what Merlin accomplished with spooky-coins.

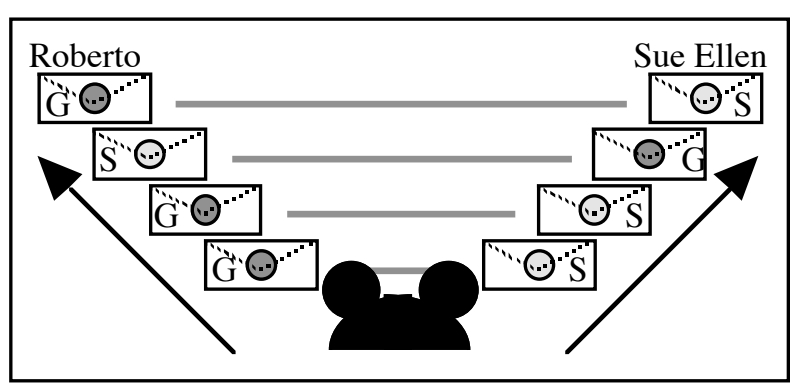

Figure 23

Alas, the apprentice's scheme cannot work. John Young, the bell ringer at the local cathedral, points this out in an unequalled line of reasoning. If Roberto and Sue Ellen randomize their rotations, then half the time their rotations match and half the time they don't. When their envelope orientations don't match, they'll get random correlations, yielding a 50\% anticorrelation, which is in fact as good (for this case) as when Merlin was stuffing spooky-coins into magicenvelopes.

However, when their envelope orientations do match, they'll get anti-correlation if the right pair of coins was stuffed in the envelopes, but random correlation if the wrong pair was stuffed. Since they get to choose how to open their envelopes after they were stuffed, the apprentice must guess which pair of coins to stuff, and therefore the best he can do is achieve a $75 \%$ anticorrelation rate for this case. This means the apprentice achieves only a $62.5 \%$ correlation rate overall. His method fails $12.5 \%$ of the time: close, but not as good as Merlin's.

\section{Faster-Than-Letter Messaging}

Finally, Merlin returns and initiates the final trial run. Now he mails two streams of magic-envelopes to each lover, one labeled A, and the other B. He instructs one of them to stack their envelopes two at a time, with one envelope from each stream, and to open them together. Whether the envelopes contain spooky-coins, or matching coins of the Realm, two of the same kind of coin will be revealed. If the envelopes contain coins of the Realm that don't match, what comes out depends on the envelope orientation. If the orientation doesn't change the type of coin revealed, then they don't match, while if it does, they match half the time. This is shown in Figure 24. 


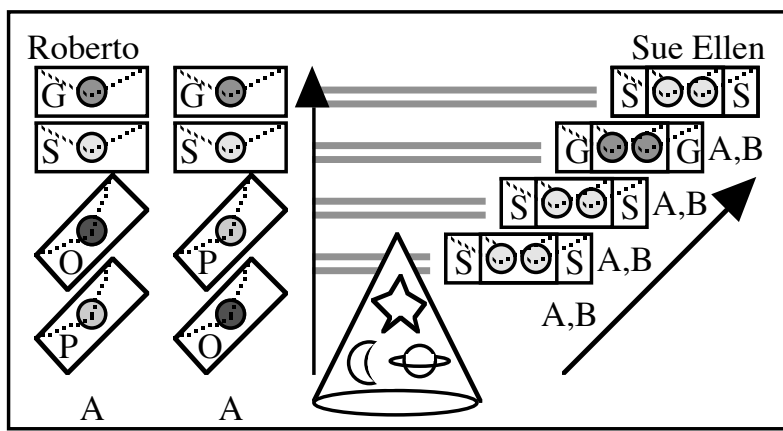

Figure 24

Now Merlin, smug in his clever scheme, asks Roberto and Sue Ellen if they can figure out how to communicate at faster-than-letter speeds. To help them out, he summarizes the rules behind the scheme.

1. Unrotated envelopes reveal a gold or silver coin.

2. Rotated envelopes reveal a platinum or osmium coin.

3. If the coin inside is congruent with the orientation of the envelope, opening the envelope does not change it. If not, the identity of the revealed coin is absolutely random.

4. When one member of a spooky-coin pair is revealed, the other "instantly" reduces to a coin of the Realm of the opposite type. If the envelopes are the same length (color), instantly means simultaneously. For different lengths, if the opened envelope was the longer, the coin in the shorter envelope will reduce a while later, while if the opened envelope was the shorter, the coin in the longer envelope reduced a while earlier.

5. Flipping an envelope inverts a coin of the Realm, and inverts the correlation of spooky-coins.

6. Stacked envelopes reveal matching coins if the coins in the envelopes are of the same type, either spooky or identical coins of the Realm. If the coins are different, stacking has no effect. Envelopes must be of the same length to stack.

Further, Merlin states that there is more than one right answer.

One way, shown in Figure 25, is for Roberto to queue up his double stream of envelopes until Sue Ellen has had a chance to open hers. She opens her envelopes in a special way, in groups of the same size called packets. To send a one-bit, she opens each $\mathrm{AB}$ pair separately, revealing randomly matching coins. To send a zero-bit, she first stacks the $\mathrm{AB}$ pair, and them opens them together revealing two matching coins. She uses the same rotation for every pair in every packet and doesn't flip any envelopes. Now Roberto opens his AB pairs separately and records them in a list. In scanning this list he should find a statistical anomaly. If he doesn't, he discards his first list and tries again, this time rotating his envelopes and making a new list. In one of these two lists, he will have found a statistical anomaly: too many matches. Refining his statistical analysis, he can discern a packet length where in some packets the matching is random, while in others, the matching is perfectly correlated. He is now receiving binary data, one bit per packet. If Sue Ellen is using any reasonable encoding scheme, Roberto can decode it with standard cryptographic tools.

Sue Ellen can now send FTL information to Roberto. To switch roles, Sue Ellen queues her envelopes for later independent opening and Roberto adopts her packetizing/stacking strategy. Now he can send her FTL information. Merlin has fulfilled his contract, setting himself up as a midpoint transmitter of a continuous dual stream of magic-envelopes containing spookycoins that provides to our two lovers a faster-than-letter communication channel in a simplex mode.

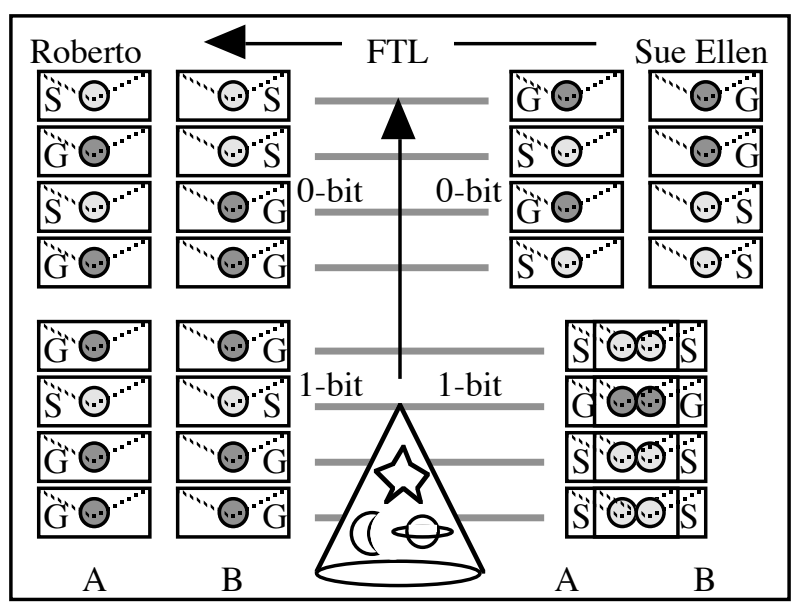

Figure 25

Since a 1-bit is encoded as randomly correlated coins via independent opening of the envelope pairs, there is a statistical chance that all the coin pairs in a packet will match, mimicking a 0-bit. This is a noise source that can be dealt with through standard error detection/correction algorithms.

\section{Einstein's Revenge}

For a time, Roberto and Sue Ellen send instant love notes back and forth. Eventually, though, they get curious about the inner workings of their FTL system, and begin to wonder if so magnificent a magic cannot be used to do other things. They send an inquiry to Mer- 
lin, who as luck would have it, is napping when the communiqué shows up, and his apprentice intercepts it. Still stung by his defeat with the classical coin fiasco, he cooks up new mischief and sends Roberto and Sue Ellen some devious directions.

"Try flipping some of your envelopes", he replies, "and who says that only one of you should be allowed to stack them?" Trustingly, they experiment along these lines.

When Roberto flips one envelope of his pair, his matching packets become anti-matching. The uncorrelated packets don't change. Now too few coins match, only $25 \%$, but it doesn't change the bits nor their detectability, just the nature of how the nonrandom packets differ from randomness. This is FTL protocol number two. Alternatively, Sue Ellen could flip one of her envelopes, but if they both do, this is back to the first FTL protocol.

Similarly, stacking, or flipping and stacking, has no significant effect on the FTL channel - unless Sue Ellen takes too long to open her envelopes. If Roberto flips and stacks too soon, then he establishes a cyclic entanglement between the two pairs of coins - and Merlin's rules generate a paradox.

The spookiness implies that each Merlin pair of coins should turn out to be of opposite type. Sue Ellen's stack implies that her pair of coins should match, but Roberto's flip and stack implies that his pair should not match. At best only three of these can be correct; one of them has to fail.

However, if Sue Ellen opens her envelopes "before" Roberto flips and stacks his, the coins in all the envelopes transition from spooky to mere coins of the Realm, and so Roberto's are now guaranteed to be different. From rule \#6, this is no problem. Therefore, the paradox can be avoided if we merely insist that the cyclic entanglement is equivalent to opening the envelopes. As in Quantum Tic-Tac-Toe, cyclic entanglement provides a measurement mechanism. Any measurement, either opening the envelopes or creating a cyclic entanglement through stacking and their spooky connections, cause the coins to collapse from spooky status to real status. All we need figure out is how the transition to real coins is to be specified.

Since there is no symmetry breaker within the cyclic entanglement, there is no a priori reason to prefer any particular coin type over another. Therefore, starting with any one coin, assume a random value for it, and follow the chain of entanglements to deduce the types of the other coins. This works fine for when there was no flipping of envelopes since the cyclic entanglement is indeterminate in these cases, as well as for even numbers of flips. However, for odd numbers of flips, the chain of entanglements involves a contradiction. There are three reasonable ways to deal with it: concentration, distribution, and limited distribution.

If concentration is the correct resolution mechanism, then one randomly selects a coin, randomly selects a real type for it to collapse to, and randomly selects a direction to proceed around the cyclic entanglement collapsing all the other coins per their entanglement relationships. The contradiction is then concentrated in just one relationship, the one between the first coin chosen and the last one in the entanglement traversal.

If distribution is the correct resolution mechanism, the contradiction is shared equally between all coin pairs with a direct entanglement relationship. This can mean that more coin types are required than have been introduced into the metaphor.

Limited distribution simply assigns earlier in time entanglements with full compliance and distributes the contradiction only among those later entanglements, which have spacelike relations with each other.

Through the above exercise, Roberto and Sue Ellen come to realize that there is a third FTL protocol. As before Roberto opens his envelope pairs separately, but now Sue Ellen always stacks hers. To encode information she flips one envelope prior to stacking for a 1-bit, and doesn't flip it for a 0-bit. Roberto receives anti correlated pairs as the 1-bit, and fully correlated pairs as the 0-bit. He no longer receives randomly correlated pairs. This means that the packets can be dispensed with in favor of simple pairs of envelopes, and the bandwidth of this protocol is an order of magnitude higher than the first two protocols, and with a zero error rate to boot.

The apprentice is still desperate to either top his master's skill, or at the very least, hamper the system Merlin has set up for Roberto and Sue Ellen. Having finally mastered spooky-coins he goes for broke and decides to use them inside red and blue envelopes. When one spooky-coin goes in a red envelope, its companion goes in a blue envelope. This time he sets up two locations from which to mail envelope pairs to Roberto and Sue Ellen. Whenever he sends a pair of red envelopes to one recipient, he sends a pair of blue envelopes to the other. He now very carefully selects his mailing loca- 
tions and strategy. A rather busy spacetime diagram of his scheme is shown in Figure 26.

From the location closer to Sue Ellen (R) he mails red envelopes to her and blue envelopes to Roberto, while from the location closer to Roberto (L) he mails red envelopes to him and blue envelopes to Sue Ellen. If the mailing locations are not too close, the recipient opening the blue envelopes becomes the sender, and the recipient opening the red envelopes becomes the receiver. So now Roberto can send FTL messages to Sue Ellen through the entangled coins mailed from near her, and Sue Ellen can send FTL messages to Roberto through the entangled coins mailed from near him. The twist is that all these messages are going backwards in time.

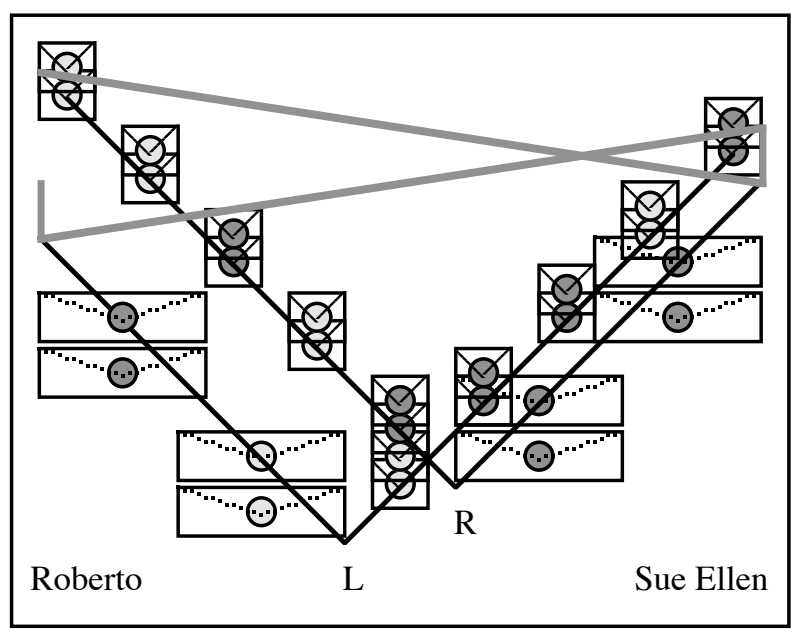

Figure 26

The spacelike backwards-in-time FTL communication links are shown in Figure 26 as the gray lines. Since it takes a little time to send and decode a packet of envelopes, vertical world lines (also in gray) are shown, indicating the reduction in the total temporal displacement achievable.

Actually, Roberto really likes this setup. Like many romantics, he often stumbles over his word choices. Finding out before he sends an FTL love message that his intended interpreted it badly, gives him a chance to correct his blunder, with Sue Ellen none the wiser. As long as she echoes even a little of what he sent, he has a chance to correct a past mistake.

As for a full resolution of the temporal paradoxes implied by this metaphor, the authors apologize. We have been unable to devise a scenario inside this metaphor that demonstrates how temporal paradox can be avoided yet put the attempt to create one to productive use.

\begin{tabular}{|l|l|}
\hline \multicolumn{1}{|c|}{$\begin{array}{c}\text { SCME } \\
\text { Metaphor }\end{array}$} & \multicolumn{1}{c|}{$\begin{array}{c}\text { Relativistic Quantum } \\
\text { Physics }\end{array}$} \\
\hline Envelope & Photon \\
\hline Coin of the Realm & Polarization \\
\hline Envelope Orientation & Polarization Axis \\
\hline Opening an Envelope & Making an Observation \\
\hline Spooky-Coins & EPR \\
\hline Sorcerer's Apprentice & Bell's Theorem \\
\hline Stacked Envelopes & Bosonic Entanglement \\
\hline Flipping an Envelope & $90^{\circ}$ Optical Rotation \\
\hline Envelope Length/Color & $\begin{array}{l}\text { Length Contracted Dop- } \\
\text { pler Shift }\end{array}$ \\
\hline “Instant" Collapse & $\begin{array}{l}\text { Symmetric Spacetime } \\
\text { Interval }\end{array}$ \\
\hline National Letter Service & c \\
\hline FTL & FTL \\
\hline
\end{tabular}

Table 2

To wrap up this section, we summarize the mapping between the spooky-coin \& magic-envelope metaphor and relativistic quantum physics in Table 2 .

\section{IMPLICATIONS}

This section considers some of the implications suggested by the two metaphors and possible areas for future research.

\section{The Game Model Metaphor of Reality}

Like a machine, a game is a rule-bound formal system. Unlike a machine, however, a game is not rule-bound all the way down to no choice. Typically, it is rulebound down to a few choices. In a machine, every effect must have a cause else the machine is broken. In a game, however, all legal moves look like uncaused effects. The rules that define legal moves create the causal connections; they prescribe what is possible, but not what specifically happens.

In classical physics, where the machine metaphor is applicable, free will could at best be illusionary. Both the past and the future are predetermined by the state of the present. The game metaphor leaves open the possibility that free will could still be a legitimate phenomenon. If it is not, neither legal systems nor religion make much sense. Similarly, neither do arguments or discourse about any subject (including this one) make any sense, for both their content and their success or failure in convincing others would, in the absence of legitimate free will, be predetermined. 
While the machine metaphor of physical reality has been indisputably successful, it fails to produce viable mental images of what is going on in quantum physics. We trust the math, but not the visualizations. With the advent of quantum games, visualizations that are consistent with the math and which produce reliable insight into what is going on, may be achievable.

\section{Extension to Other Games}

The basic ideas behind Quantum Tic-Tac-Toe can be extended to other games, perhaps most games. The resulting complexity, however, is likely to make them relatively unplayable. Despite this, the attempt may offer additional insights into the nature of quantum reality.

In such quantum games, a player indicates two or more possible legal moves on each turn. A player's mixedstate move may be different moves of the same piece, or it may involve moving different pieces. Both are valid branches down the classical game tree. A major issue to consider is what constitutes an entanglement between mixed state moves and whether it leads to reasonable measurement events. Since pieces don't move in Tic-Tac-Toe while they do move in many other games, measurement events may work a little differently.

Since Quantum Tic-Tac-Toe does not offer a metaphor for conjugate quantities, the quantization of some other game may be necessary in order to fully extend the game paradigm. Perhaps the distinction between games where pieces are merely placed (Tic-Tac-Toe, Go, etc.) and those where they move from square to square (chess, checkers, etc.) will offer gains in this area.

\section{Coupled Alternative Realities}

In the quantum game metaphor, the multiple classical games in simultaneous play take on the role of alternative realities. Unlike Everett's Many Worlds Hypothesis, which includes the entire game tree from the Big Bang onward, the number of these realities is more limited. Also, the number in existence, instead of growing exponentially, may merely fluctuate around a relatively stable value, as entanglements alternatively grow and collapse.

From a technologist's point of view, the idea that entire universes can be branched into existence in quantum entanglements and snuffed out in quantum collapses, offers a unique cause and effect mechanism. What would have happened in some universes that have evaporated has successfully influenced what actually happened in the ones that persisted. A cause from one universe to another is instantaneous, unmediated, and undiminished with distance. It is a totally different causal connection than mechanics or fields, and has the potential to fit NASA's criteria for breakthrough propulsion physics. It does seem to be a viable mechanism to permit faster-than-light communications.

\section{World Views}

A game metaphor of reality invites speculation on who or what are the Players. We'll capitalize "Players" in this section to emphasize that they are outside the rules. Being that they are outside the rules, how much science can say about them is an open question. The assumption that they are real, however, does allow consideration of a potentially important distinction about natural law.

The appropriate analogy here is to suppose that you have entered a room where many expert Players are engaged in playing a game with which you are unfamiliar. The Players permit observation, but will not explain the rules. A studious observer stands a reasonable chance of figuring out the rules to the game just by watching. This is like the situation of the scientist who looks out into the universe and tries to figure out the rules that govern it by keen observation, insight, analysis, and imagination. It is by no means a given that the Universe should be understandable to us, but we seem to live in a reality where this is so.

However, what we cannot do, even in principle, is discern rules from strategies. If the Players are masters, intent on winning and playing well, then the patterns observers witness cannot be identified as rules or strategies. It is not a rule that the first move in a chess game must be a central pawn, but no games at the grand master level start in any other way. While it was reasonable to expect that the rules of a machine were fixed at its genesis, and it is just as reasonable to expect that the rules of a game are fixed and immutable, it is not so clear whether we should regard strategies as fixed or variable.

If the strategies do not change over the course of a complete game, or if play is entirely random, or if the Players' choices are themselves determined by immutable rules, then there is no practical distinction between rules and strategies. Aesthetics might suggest a difference, but drawing a scientific distinction is problematical.

In game theory, multi-player games yield radically different strategies than two player games. Multi-player games tend to degenerate through alliances into two- 
player games with a concomitant shift in strategies. If the strategies can change over time, either smoothly or discontinuously, then some of what we now regard as laws of nature may lose their universal applicability.

\section{SUMmaRY \& CONCLUSIONS}

The major result of this effort is the applicability of quantized games to suggest a new paradigm of what quantum physics "means". Striking at the core of physics, quantum games offer up measurement mechanisms that should be testable.

Implicit in this approach is an explanation of spooky action at a distance as an inter-universe causal connection. When quantum rules are interpreted as meta-rules for a classical structure, a quantum state implies a multiplicity of simultaneously existing classical realities. As quantum systems subsequently entangle and collapse, multiple realities branch into existence, and evaporate out of existence, such that realities that might have existed influence the ones that do.

This influence constitutes an inter-universe causal connection which is immediate, unmediated, and undiminished by distance. It implies that faster-than-light signaling will actually be possible. While the metaphors do not well address the issues of temporal paradox, the companion paper of Reference 2 does so in some detail.

It is our hope, that Quantum Tic-Tac-Toe will find a place in the classroom as an engaging introduction to the counter intuitive nature of quantum physics. If it can aid the student in coming to grips with the nature of quantum reality, it will have well served its purposes.

\section{REFERENCES}

1. Bell, John S., Speakable and Unspeakable in Quantum Mechanics, Chapter 3, "The Moral Aspect of Quantum Mechanics", Cambridge University Press, New York, NY, 1993, p 26.

2. Goff, A., D. Lehmann, J. Siegel, "Relativistically Consistent Faster-than-Light (FTL) Communication Channel Using Self-Referential Quantum States", AIAA Paper 2002-4093, $38^{\text {th }}$ Joint Propulsion Conference, Indianapolis, IN, July 7-10, 2002.

3. Wheeler, J. A., "The Past and Delayed Choice Double Slit Experiment", in Mathematical Foundations of Quantum Theory: Proceedings of the New Orleans Conference on the Mathematical Foundations of Quantum Theory, A. R. Marlow, ed., Academic Press, New York, NY, 1978.

4. De Witt, B. S. and R. D. Graham, (eds.) (1973). The Many-Worlds Interpretation of Quantum Mechanics, Princeton University Press.

5. Von Neumann, John, Oskar Morgenstern, Theory of Games and Economic Behavior, Princeton University Press, 1941.

6. Penrose, Roger, The Emperor's New Mind, Oxford University Press, New York, NY, 1989, p 343.

7. Smolin, Lee, Three Roads to Quantum Gravity, Basic Books, New York, NY, 2001, p 197.

8. Ross, Hugh, The Fingerprint of God, Promise Press, Orange, CA, 1989.

9. Herbert, Nick, Quantum Reality, Beyond the New Physics, Doubleday, New York, NY, 1985, p 171.

10. Einstein A, N. Rosen, and B. Podolsky, "Can Quantum-Mechanical Description of Physical Reality Be Considered Complete?", Physical Review 47 777 (1935).

11. Bell, John S., "On the Problem of Hidden Variables in Quantum Mechanics", Reviews of Modern Physics 38447 (1966). 
Notes

\begin{tabular}{|c|c|}
\hline $\mathbf{X}$ & $\mathbf{O}$ \\
\hline--- & --- \\
\hline--- & --- \\
\hline--- & --- \\
\hline--- & --- \\
\hline--- & \\
\hline
\end{tabular}
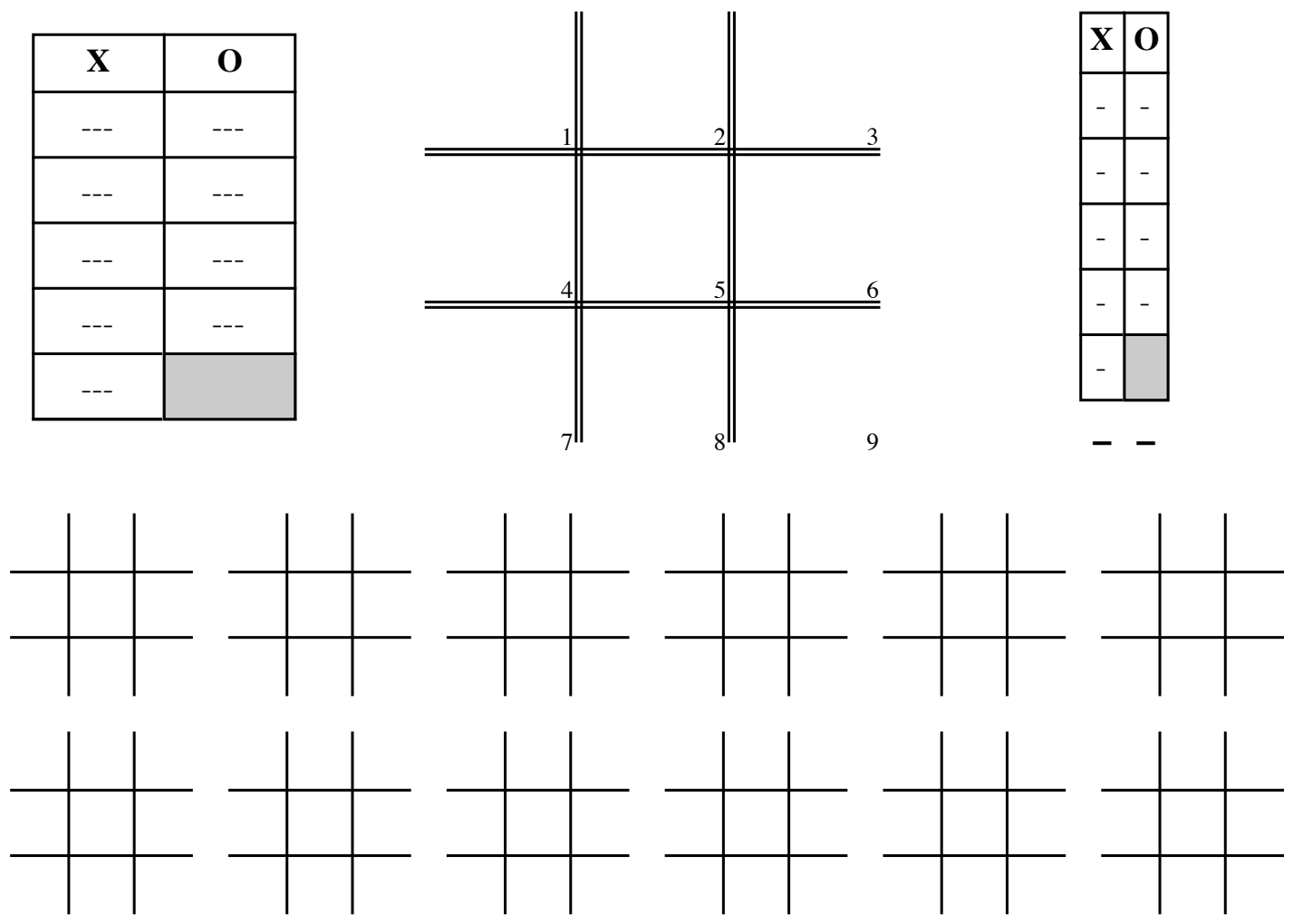

\begin{tabular}{|c|c|}
\hline $\mathbf{X}$ & $\mathbf{O}$ \\
\hline--- & --- \\
\hline--- & --- \\
\hline--- & --- \\
\hline--- & --- \\
\hline--- & \\
\hline
\end{tabular}
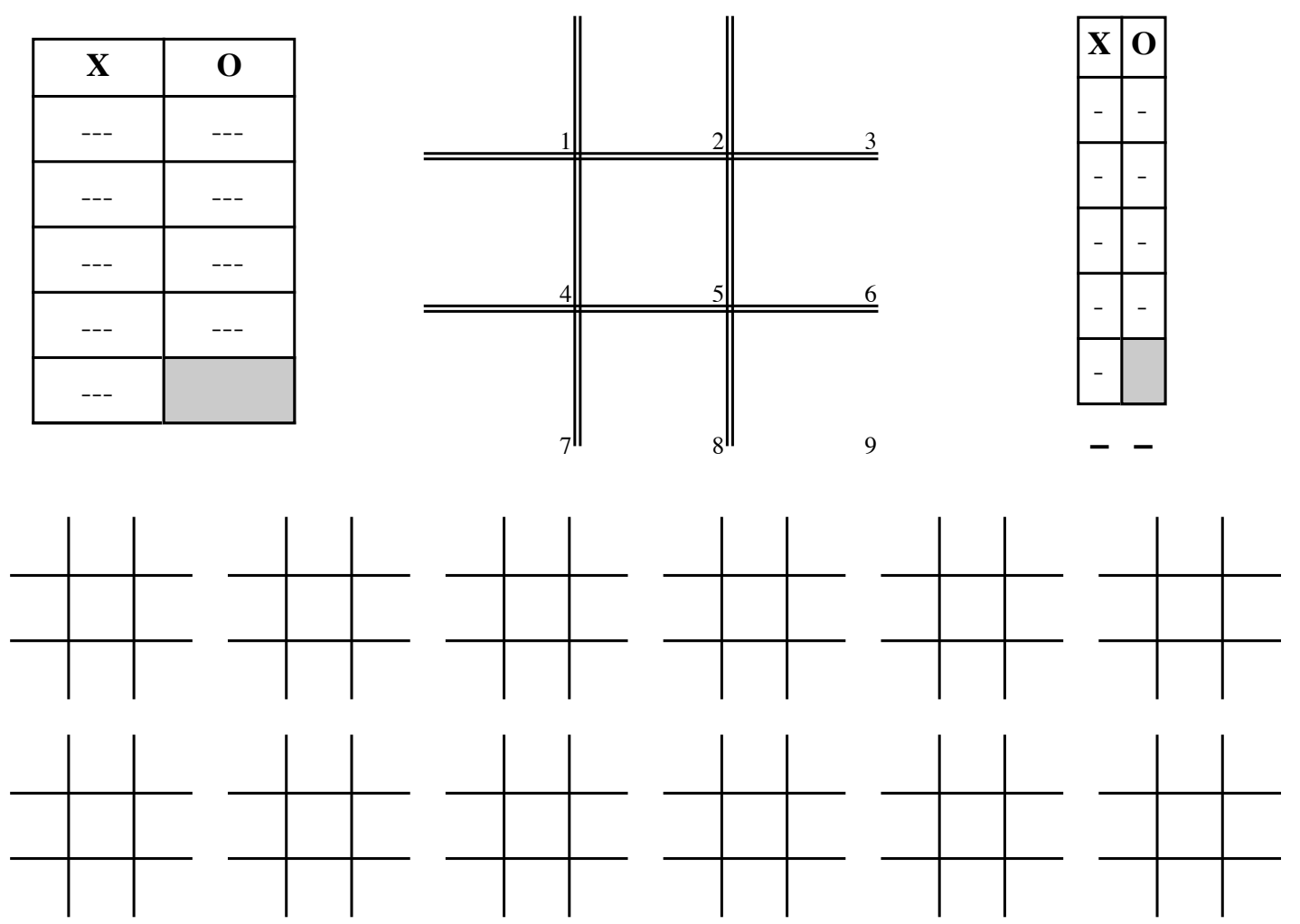


\section{Quantum Tic-Tac-Toe}

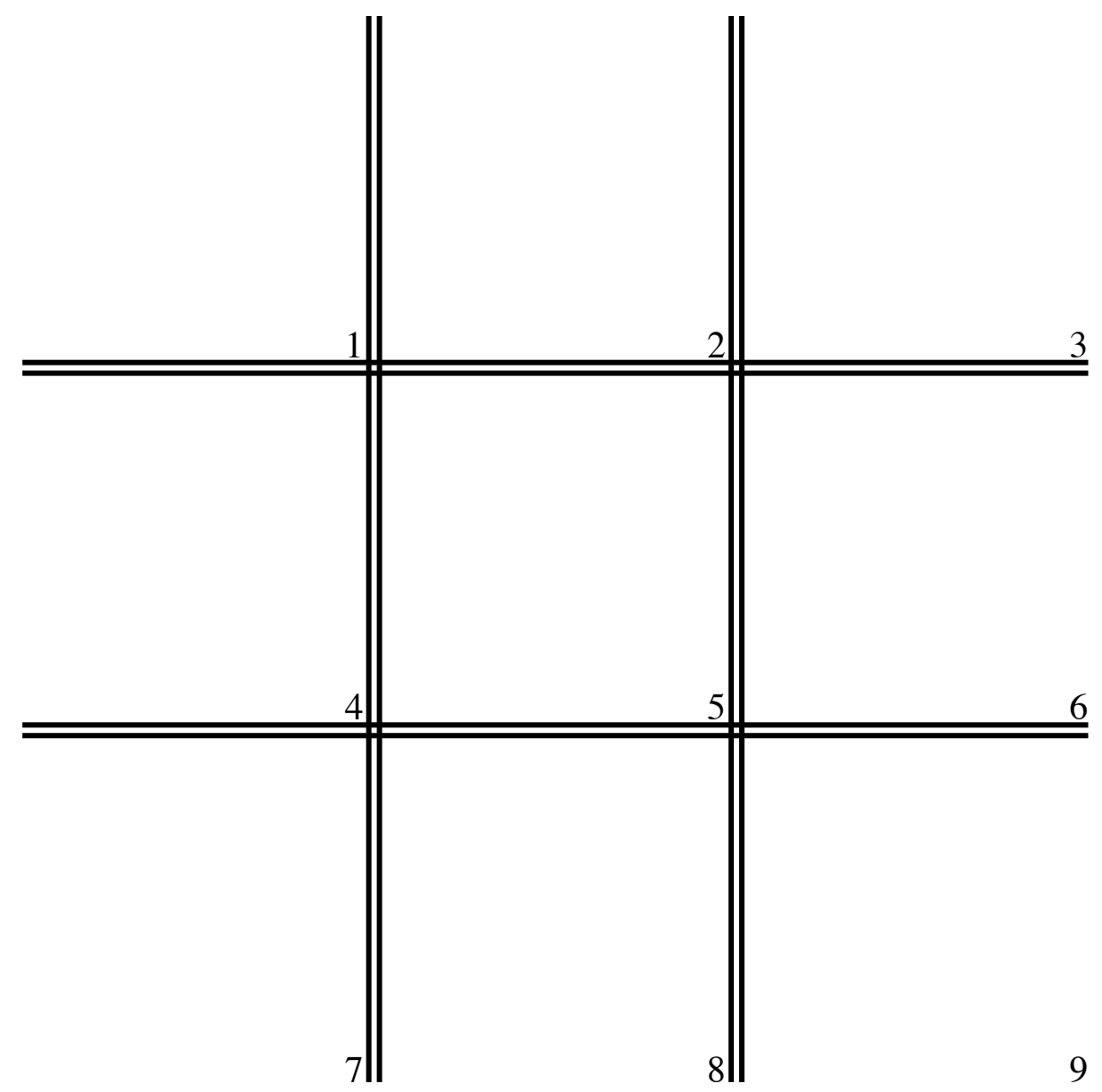

The rules for Quantum Tic-Tac-Toe are the same as for Classical Tic-Tac-Toe except...

1. Players take turns making quantum moves with X going first. Quantum marks are pairs of X's or O's subscripted with the number of the move. In a quantum move, two different squares are marked at once - a quantum move looks like a classical move that is half in one square and half in another.

2. Quantum moves may entangle by sharing squares. An entanglement arises when two or more quantum moves share a square.

3 . On a cyclic entanglement, the other player chooses the collapse to classical moves. Indicate the kept halves, one per square, and eliminate the others. A cyclic entanglement occurs whenever there is a path from one square back to itself. In acollapse the quantum moves are replaced with classical moves.

4. First player to get 3 in a row of classical moves wins. For simultaneous 3 -rows, the earlier in time gets a full point, the later a half point. Anearlier in time 3-row has the lower largest subscript. 